\title{
Classical and Quantum Shortcuts to Adiabaticity for Scale-Invariant Driving
}

\author{
Sebastian Deffner, ${ }^{1}$ Christopher Jarzynski, ${ }^{1}$ and Adolfo del Campo ${ }^{2,3}$ \\ ${ }^{1}$ Department of Chemistry and Biochemistry and Institute for Physical Science and Technology, \\ University of Maryland, College Park, Maryland 20742, USA \\ ${ }^{2}$ Theoretical Division, Los Alamos National Laboratory, Los Alamos, New Mexico 87545, USA \\ ${ }^{3}$ Center for Nonlinear Studies, Los Alamos National Laboratory, Los Alamos, New Mexico 87545, USA
}

(Received 6 January 2014; published 22 April 2014)

\begin{abstract}
A shortcut to adiabaticity is a driving protocol that reproduces in a short time the same final state that would result from an adiabatic, infinitely slow process. A powerful technique to engineer such shortcuts relies on the use of auxiliary counterdiabatic fields. Determining the explicit form of the required fields has generally proven to be complicated. We present explicit counterdiabatic driving protocols for scale-invariant dynamical processes, which describe, for instance, expansion and transport. To this end, we use the formalism of generating functions and unify previous approaches independently developed in classical and quantum studies. The resulting framework is applied to the design of shortcuts to adiabaticity for a large class of classical and quantum, single-particle, nonlinear, and many-body systems.
\end{abstract}

DOI: 10.1103/PhysRevX.4.021013

\section{INTRODUCTION}

Modern research in nanoengineering develops increasingly small devices that operate in a regime described by effective classical dynamics [1,2] or quantum mechanics $[3,4]$. Achieving a fast coherent control with high fidelity $[5,6]$ is a ubiquitous goal shared by a variety of fields and technologies, including quantum sensing and metrology [7], finite-time thermodynamics [8], quantum simulation [9], and adiabatic quantum computation [10]. The quantum adiabatic theorem [11], however, appears as a no-go theorem for excitation-free ultrafast processes. As a result, an increasing amount of theoretical and experimental research is targeting the design of shortcuts to adiabaticity (STA), i.e., nonadiabatic processes that reproduce in a finite time the same final state that would result from an adiabatic, infinitely slow protocol [12].

A variety of techniques has been developed to engineer STA: The use of dynamical invariants $[13,14]$, the inversion of scaling laws [15,16], the fast-forward technique [17-19], and counterdiabatic driving, also known as transitionless quantum driving [20-22]. Among these techniques, counterdiabatic driving (CD) is unique in that it drives the dynamics precisely through the adiabatic manifold of the system Hamiltonian. In addition, it enjoys a wide applicability. In its original formulation [20-22], one considers a time-dependent Hamiltonian $\hat{H}_{0}(t)$ with instantaneous

Published by the American Physical Society under the terms of the Creative Commons Attribution 3.0 License. Further distribution of this work must maintain attribution to the author(s) and the published article's title, journal citation, and DOI.
Subject Areas: Interdisciplinary Physics, Quantum Physics

eigenvalues $\left\{\varepsilon_{n}(t)\right\}$ and eigenstates $\{|n(t)\rangle\}$. In the limit of infinitely slow variation of $\hat{H}_{0}(t)$, a solution of the dynamics is given by

$$
\left|\psi_{n}(t)\right\rangle=e^{-(i / \hbar) \int_{0}^{t} d s \varepsilon_{n}(s)-\int_{0}^{t} d s\left\langle n \mid \partial_{s} n\right\rangle}|n(t)\rangle .
$$

In this adiabatic limit, no transitions between eigenstates occur [11], and each eigenstate acquires a time-dependent phase that can be separated into a dynamical and a geometric contribution [23], represented by the two terms inside the exponential in the above expression.

Now, consider a nonadiabatic Hamiltonian $\hat{H}_{0}(t)$. In the CD paradigm, a corresponding Hamiltonian $\hat{H}(t)$ is constructed, such that the adiabatic approximation associated with $\hat{H}_{0}(t)$ [Eq. (1)] is an exact solution of the dynamics generated by $\hat{H}(t)$ under the time-dependent Schrödinger equation. Writing the time-evolution operator as $\hat{U}(t)=\sum_{n}\left|\psi_{n}(t)\right\rangle\langle n(0)|$, one arrives at an explicit expression for $\hat{H}(t)$ [20-22]:

$$
\hat{H}=\hat{H}_{0}+\hat{H}_{1}=\hat{H}_{0}+i \hbar \sum_{n}\left(\left|\partial_{t} n\right\rangle\left\langle n\left|-\left\langle n \mid \partial_{t} n\right\rangle\right| n\right\rangle\langle n|\right) .
$$

Here, the auxiliary CD Hamiltonian $\hat{H}_{1}(t)$ enforces evolution along the adiabatic manifold of $\hat{H}_{0}(t)$ : If a system is prepared in an eigenstate $|n(0)\rangle$ of $\hat{H}_{0}(0)$ and subsequently evolves under $\hat{H}(t)$, then the term $\hat{H}_{1}(t)$ effectively suppresses the nonadiabatic transitions out of $|n(t)\rangle$ that would arise in the absence of this term. Note that the evolution is nonadiabatic with respect to the full $\mathrm{CD}$ Hamiltonian $\hat{H}=\hat{H}_{0}+\hat{H}_{1}$. 
The CD Hamiltonian $\hat{H}(t)$ has been the object of intense study. It was found that the higher the speed of evolution, the larger is the intensity of the required auxiliary CD field $[24,25]$. Experimental demonstrations of driving protocols inspired by the $\mathrm{CD}$ technique have recently been reported in single two-level systems [26,27]. In the many-body case, $\hat{H}_{1}(t)$ generally includes nonlocal and multibody interactions [25,28]. Local driving protocols can be derived for unitarily equivalent Hamiltonians [28], an approach that has also proven useful in single-particle systems $[12,26,27,29]$.

However, the computation of the auxiliary term $\hat{H}_{1}(t)$ requires knowledge of the spectral properties of the instantaneous system Hamiltonian $\hat{H}_{0}(t)$ at all times. This constraint has limited the range of applicability of the method to the control of few-level systems [20-22] and noninteracting matter waves in time-dependent harmonic traps [30-32].

Recently, a classical analogue of CD was proposed, namely, dissipationless classical driving [33]. Here, for a time-dependent classical Hamiltonian $H_{0}(q, p, t)$, one seeks an auxiliary term $H_{1}(q, p, t)$ such that under the Hamiltonian dynamics generated by $H=H_{0}+H_{1}$, the classical adiabatic invariant of $H_{0}$ is conserved exactly. For systems with one degree of freedom, an explicit solution of this problem, analogous to Eq. (1) above, was obtained [see Eq. (32) of Ref. [33]]. Moreover, it was argued that this classical solution can be useful in constructing the quantal CD Hamiltonian $\hat{H}(t)$, bypassing the spectral decomposition of $\hat{H}_{0}(t)$. This idea was illustrated for arbitrary power-law traps (including the particle in a box as a limiting case), for which simple expressions for $\hat{H}_{1}(t)$ in terms of position and momentum operators were obtained and quantized. Further progress was achieved using scaling laws in expansions and compressions for a wide variety of single-particle, nonlinear, and manybody quantum systems [28].

Our aim in this paper is to find an experimentally realizable CD Hamiltonian (2) for scale-invariant processes, without using the explicit spectral decomposition of $\hat{H}_{0}(t)$. Scale-invariant driving is generated by transformations of $\hat{H}_{0}(t)$ for which the density profile (and all local correlations in real space) is preserved up to scaling and translation. Using this property, we start with a single quantum particle in a one-dimensional potential, from which we will develop a general framework to find local CD protocols for multiparticle quantum systems, obeying both linear and nonlinear dynamics. We will use methods from classical Hamiltonian dynamics, namely, the formalism of generating functions, to treat dissipationless classical driving by the same means. Our approach also allows one to treat arbitrary external potentials, beyond the validity of perturbation theory.

The paper is organized as follows: We will begin in Sec. II by deriving an expression for the CD Hamiltonian (2) for the scale-invariant driving of a quantum system with one degree of freedom. Section III is dedicated to classical Hamiltonian dynamics, in which the classical version of $\hat{H}_{1}$ can be rewritten in a local form using linear canonical transformations and the formalism of generating functions. These findings will be generalized and applied in Sec. IV to a broad family of many-body quantum systems. Specific protocols for arbitrary trapping potentials will be discussed in Sec. V. Section VI is dedicated to nonlinear systems, with emphasis in mean-field theories. In Sec. VII, we will discuss the relation of CD to more general scaling laws, before we explicitly engineer STA in Sec. VIII. We close with a summary and discussion in Sec. IX.

\section{COUNTERDIABATIC HAMILTONIAN FOR SCALE-INVARIANT DRIVING}

Generally, it appears to be hardly feasible to find closedform expressions, i.e., expressions that do not depend on the full spectral decomposition of $\hat{H}_{0}(t)$, for the auxiliary term in the CD Hamiltonian $\hat{H}(t)(2)$. Recently, it has been shown that scale invariance greatly facilitates this task for processes that describe self-similar expansions and compressions in a time-dependent trap [28], including the family of power-law potentials as a special case [33]. More generally, scale-invariant driving refers to transformations of the system Hamiltonian associated with a set of external control parameters $\lambda(t)=\left[\lambda_{1}(t), \ldots, \lambda_{n}(t)\right]$ that can be absorbed by scaling of coordinates, time, energy, and possibly other variables to rewrite the transformed Hamiltonian in its original form up to a multiplicative factor. If only the potential term $U[q, \lambda(t)]$ is modulated, its overall shape does not change under $\lambda(0) \rightarrow \lambda(t)$. For the time being, we focus on a quantum system with a single degree of freedom

$$
\hat{H}_{0}(t)=\frac{p^{2}}{2 m}+U[q, \lambda(t)]=\frac{p^{2}}{2 m}+\frac{1}{\gamma^{2}} U_{0}\left(\frac{q-f}{\gamma}\right),
$$

where $\boldsymbol{\lambda}=(\gamma, f)$ and $U_{0}(q)=U[q, \boldsymbol{\lambda}(0)]$. Note that generally, $\gamma=\gamma(t)$ and $f=f(t)$ are both allowed to be time dependent, but we assume that they are independent of each other. This time dependence encompasses transport processes $[\gamma(t)=1]$, dilations [such as an expansion or compression, with $f(t)=0$ ], and combined dynamics, which are the focus of our attention and elements of the dynamical group of the system Hamiltonian, the universal covering group of $S U(1,1), \overline{S U(1,1)}$ [34].

Our goal is to rewrite the auxiliary term $\hat{H}_{1}(t)$ [Eq. (2)] into a form that does not rely on the spectral decomposition of $\hat{H}_{0}(t)$. Let $\psi_{n}^{0}(q)=\langle n \mid q\rangle$ be an eigenfunction of the Hamiltonian $\hat{H}_{0}(\gamma=1, f=0)$; then, $\psi_{n}(q, \gamma, f)=$ $\alpha(\gamma) \psi_{n}^{0}[(q-f) / \gamma]$ is an eigenfunction of $\hat{H}_{0}(\gamma, f)$, where $\alpha(\gamma)=1 / \sqrt{\gamma}$ is a normalization constant. The proof of this statement can be found in Appendix A.

Now, we want to use this symmetry to simplify $\hat{H}_{1}(t)$ in Eq. (2). We have 


$$
\hat{H}_{1}(t)=i \hbar \dot{\lambda} \cdot \sum_{m}\left(\left|\nabla_{\lambda} m\right\rangle\left\langle m\left|-\left\langle m \mid \nabla_{\lambda} m\right\rangle\right| m\right\rangle\langle m|\right),
$$

which reads in space representation

$$
\begin{aligned}
\hat{H}_{1}(t)= & i \hbar \dot{\lambda} \cdot \sum_{m} \int d q|q\rangle \nabla_{\lambda} \psi_{m}(q, \lambda)\langle m| \\
& -i \hbar \dot{\lambda} \cdot \sum_{m} \int d q\langle m \mid q\rangle \nabla_{\lambda} \psi_{m}(q, \lambda)|m\rangle\langle m| .
\end{aligned}
$$

To simplify this expression, we note that

$$
\begin{aligned}
& \nabla_{\lambda} \psi_{n}(q, \lambda) \\
& \quad=\left(\frac{\alpha^{\prime}(\gamma)}{\alpha(\gamma)} \psi_{n}(q, \gamma)-\frac{q-f}{\gamma} \partial_{q} \psi_{n}(q, \gamma),-\partial_{q} \psi_{n}(q, \gamma)\right) .
\end{aligned}
$$

For the sake of clarity, let us treat both terms of $\hat{H}_{1}(t)$ in Eq. (5) separately. We obtain for the first term

$$
\begin{aligned}
i \hbar \dot{\lambda} \cdot & \sum_{m} \int d q|q\rangle \nabla_{\lambda} \psi_{m}(q, \lambda)\langle m| \\
& =\frac{\dot{\gamma}}{\gamma}(q-f) p+i \hbar \dot{\gamma} \frac{\alpha^{\prime}(\gamma)}{\alpha(\gamma)}+\dot{f} p,
\end{aligned}
$$

while the second term reduces to

$$
\begin{aligned}
& -i \hbar \dot{\lambda} \cdot \sum_{m} \int d q\langle m \mid q\rangle \nabla_{\lambda} \psi_{m}(q, \lambda)|m\rangle\langle m| \\
& =-\frac{i \hbar \dot{\gamma}}{2 \gamma}-i \hbar \dot{\gamma} \frac{\alpha^{\prime}(\gamma)}{\alpha(\gamma)} .
\end{aligned}
$$

Note that the second component of $\nabla_{\lambda} \psi_{n}(q, \lambda)$ does not contribute, since the wave function vanishes at infinity due to normalizability. In conclusion, we obtain the explicit expression of the auxiliary CD Hamiltonian

$$
\hat{H}_{1}(t)=\frac{\dot{\gamma}}{2 \gamma}[(q-f) p+p(q-f)]+\dot{f} p,
$$

where we use $[q-f, p]=i \hbar$. Notice that $\hat{H}_{1}(t)$ in Eq. (9) is of the general form $\hat{H}_{1} \propto(q p+p q)$, which was found for a time-dependent harmonic trap [30] and more generally in Refs. [28,33] for the class of potentials

$$
U[q, \gamma(t)]=\frac{A}{\gamma^{2}}\left(\frac{q}{\gamma}\right)^{b}
$$

where $b \in\{2,4,6, \ldots\}$ and $A>0$. See also Refs. [33,35] for a discussion of the limiting case $b \rightarrow \infty$, that of a boxlike confinement. Obviously, this class [Eq. (10)] belongs to the more general scale-invariant potentials introduced above in Eq. (3).
Equation (9) is our first main result. For all driving protocols under which the original Hamiltonian $\hat{H}_{0}(t)$ is scale invariant, i.e., where the time-dependent potential is of the form of Eq. (3), the auxiliary term $\hat{H}_{1}(t)$ takes the closed form [Eq. (9)]. In particular, $\hat{H}_{1}(t)$ is independent of the explicit energy eigenfunctions and only depends on the anticommutator $\hat{H}_{1} \propto\{q, p\}=q p+p q$, the generator of dilations. As a result, $C D$ applies not only to single eigenstates but also to nonstationary quantum superpositions and mixed states. However, the expression (9) is still not particularly practical, as nonlocal Hamiltonians [36] are hard to realize in the laboratory. We continue our analysis by explicitly constructing coordinate transformations that allow us to write $\hat{H}_{1}(t)$ in local form, i.e., where $\hat{H}_{1}(t)$ depends only on position. In order to do so, we will use the classical version of $\mathrm{CD}$ as a guide.

\section{SCALE-INVARIANT DRIVING: A CASE FOR GENERATING FUNCTIONS}

We now turn to dissipationless classical driving [32,33], the classical analogue of quantum counterdiabatic driving. For scale-invariant Hamiltonians, the connection between the quantum and classical cases is particularly close, and the corresponding auxiliary CD terms $\hat{H}_{1}(t)$ and $H_{1}(t)$ are essentially identical, up to quantization.

In complete analogy with the quantum case, we consider a classical Hamiltonian with one degree of freedom

$$
H_{0}(z, t)=H_{0}[z ; \lambda(t)]=\frac{p^{2}}{2 m}+U[q, \lambda(t)],
$$

where $z=(q, p)$ is a point in phase space. The classical adiabatic invariant is given by

$$
\omega(z, \lambda)=\Omega\left[H_{0}(z, \lambda), \lambda\right]
$$

where

$$
\Omega(E, \lambda)=\int d z \Theta\left[E-H_{0}(z, \lambda)\right]
$$

is the volume of phase space enclosed by the energy shell $E$ of $H_{0}(z, \lambda)$. In the adiabatic limit, $\omega[z(t), \lambda(t)]$ remains constant along a Hamiltonian trajectory $z(t)$ evolving under $H_{0}[z, \lambda(t)]$, just as the quantum number $n$ remains constant in the quantum case. We now consider nonadiabatic driving of the parameters $\lambda(t)$, and we seek an auxiliary CD term

$$
H_{1}(z, t)=\dot{\lambda} \cdot \xi[z, \lambda(t)],
$$

resembling Eq. (4), such that $\omega$ remains constant at an arbitrary driving speed, for any trajectory evolving under the Hamiltonian $H(z, t)=H_{0}[z, \lambda(t)]+H_{1}(z, t)$.

It is useful to picture dissipationless driving in terms of an ensemble of trajectories evolving under $H(z, t)$, with initial conditions sampled from an energy shell $E(0)$ of 
$H_{0}[z ; \lambda(0)]$. Since the value of $\omega$ is preserved for every trajectory in this ensemble, at any later time $t>0$, these trajectories populate a single energy shell $E(t)$ of $H_{0}[z ; \lambda(t)]$, determined by the condition $\Omega[E(t), \lambda(t)]=$ $\Omega[E(0), \lambda(0)]$, which defines the adiabatic energy shell.

As discussed in Ref. [33], it is useful to view $\xi(z, \lambda)$ as a generator of infinitesimal transformations $z \rightarrow z+d z$, with

$$
d z=d \lambda \cdot\{z, \xi\},
$$

where $\{A, B\}=\partial_{q} A \cdot \partial_{p} B-\partial_{p} A \cdot \partial_{q} B$ is the Poisson bracket. Equation (15) provides a rule for converting a small change of parameters $d \boldsymbol{\lambda}$ into a small displacement in phase space $d z$. In order to achieve dissipationless classical driving, the energy shells of $H_{0}(\lambda)$ must be mapped, under Eq. (15), onto those of $H_{0}(\lambda+d \lambda)$, with

$$
\omega(z+d z, \lambda+d \lambda)=\omega(z, \lambda) .
$$

When this condition is satisfied, the term $H_{1}=\dot{\boldsymbol{\lambda}} \cdot \boldsymbol{\xi}$ provides precisely the counterdiabatic driving required to preserve the value of $\omega$. Thus, to construct the CD Hamiltonian, we must find the function $\xi(z, \lambda)$ that generates infinitesimal deformations of the adiabatic energy shell, as per Eqs. (15) and (16).

Our scale-invariant Hamiltonian

$$
H_{0}(z ; \gamma, f)=\frac{p^{2}}{2 m}+\frac{1}{\gamma^{2}} U_{0}\left(\frac{q-f}{\gamma}\right)
$$

satisfies

$$
\begin{aligned}
H_{0}(q+a, p ; \gamma, f+a) & =H_{0}(q, p ; \gamma, f), \\
H_{0}\left(r q, \frac{p}{r} ; r \gamma, r f\right) & =\frac{1}{r^{2}} H_{0}(q, p ; \gamma, f), \\
\Omega(E, \gamma, f) & =\Omega\left(\gamma^{2} E, 1,0\right),
\end{aligned}
$$

for any real $a$ and positive $r$. Using these properties, we can verify by direct substitution that the canonical mapping

$$
(q, p) \rightarrow\left(q+d f+\frac{d \gamma}{\gamma}(q-f), p-\frac{d \gamma}{\gamma} p\right)
$$

satisfies Eq. (16). The change $f \rightarrow f+d f$ produces a coordinate translation, while under the change $\gamma \rightarrow \gamma+d \gamma$, the adiabatic energy shell is stretched along the coordinate $q-f$ and compressed along the momentum $p$. The infinitesimal transformation (19) is generated by

$$
\xi_{\gamma}=\frac{(q-f) p}{\gamma}, \quad \xi_{f}=p
$$

as verified by substitution into Eq. (15), with $\lambda=(\gamma, f)$ and $\boldsymbol{\xi}=\left(\xi_{\gamma}, \xi_{f}\right)$. Combining Eqs. (14) and (20), we arrive at

$$
H_{1}(z, t)=\frac{\dot{\gamma}}{\gamma}(q-f) p+\dot{f} p
$$

the classical counterpart of Eq. (9). With this auxiliary term, the value of $\omega[z(t), \lambda(t)]$ remains constant along a trajectory evolving under the Hamiltonian $H=H_{0}+H_{1}$, for any protocol $\lambda(t)$. To illustrate this general result, we derive $H_{1}(t)$ for an analytically solvable example, namely, the parametric Morse oscillator in Appendix B.

Equation (21) gives us a nonlocal CD Hamiltonian that accomplishes dissipationless classical driving. Our goal now is to find a coordinate transformation mapping $(q, p)$ to a set of new variables $(\bar{q}, \bar{p})$, and a corresponding Hamiltonian $\bar{H}(\bar{q}, \bar{p}, t)$ whose dynamics (in $q$ space) is identical to that under $H(q, p, t)$, and for which $\bar{H}(\bar{q}, \bar{p}, t)$ is local; i.e., it is the sum of a kinetic energy term proportional to $\bar{p}^{2}$ and a function $\bar{U}(\bar{q}, t)$. In classical mechanics, this type of problem can be elegantly solved using the formalism of generating functions [37].

We briefly recall the main idea. Let $h_{1}\left(q_{1}, p_{1}, t\right)$ be a time-dependent Hamiltonian, written in terms of coordinates $\left(q_{1}, p_{1}\right)$ in a two-dimensional phase space. Now, consider new coordinates $\left(q_{2}, p_{2}\right)$ that are related to $\left(q_{1}, p_{1}\right)$ by a time-dependent canonical transformation:

$$
q_{2}=q_{2}\left(q_{1}, p_{1}, t\right), \quad p_{2}=p_{2}\left(q_{1}, p_{1}, t\right) .
$$

Since canonical transformations are invertible, we can alternatively express the "old" coordinates $\left(q_{1}, p_{1}\right)$ as functions of the "new" ones $\left(q_{2}, p_{2}\right)$. If a function $F\left(q_{1}, p_{2}, t\right)$ can be constructed such that the relationship between the two coordinate sets is given by

$$
p_{1}=\frac{\partial F}{\partial q_{1}}, \quad q_{2}=\frac{\partial F}{\partial p_{2}},
$$

then $F\left(q_{1}, p_{2}, t\right)$ is called a generating function of a typetwo canonical transformation [37]. We can then define a Hamiltonian

$$
h_{2}\left(q_{2}, p_{2}, t\right)=h_{1}+\frac{\partial F}{\partial t}
$$

that generates trajectories equivalent to those of $h_{1}\left(q_{1}, p_{1}, t\right)$. Specifically solutions to the equations

$$
\dot{q}_{1}=\frac{\partial h_{1}}{\partial p_{1}}, \quad \dot{p}_{1}=-\frac{\partial h_{1}}{\partial q_{1}},
$$

when rewritten in the new coordinates, become solutions to

$$
\dot{q}_{2}=\frac{\partial h_{2}}{\partial p_{2}}, \quad \dot{p}_{2}=-\frac{\partial h_{2}}{\partial q_{2}} .
$$

The function $F\left(q_{1}, p_{2}, t\right)$ thus encodes the transformation of both the variables (23) and the Hamiltonian (24). 
In what follows, we shall apply this approach to three different sets of coordinates related by canonical transformations. The $\mathrm{CD}$ Hamiltonian for scale-invariant dynamics with the nonlocal term [Eq. (21)] reads

$$
H(q, p, t)=\frac{p^{2}}{2 m}+\frac{1}{\gamma^{2}} U_{0}\left(\frac{q-f}{\gamma}\right)+\frac{\dot{\gamma}}{\gamma}(q-f) p+\dot{f} p .
$$

Now, we define a type-two generating function

$$
F(q, \bar{p}, t)=q(\bar{p}-m \dot{f})-\frac{m}{2} \frac{\dot{\gamma}}{\gamma}(q-f)^{2}+\frac{m}{2} \int_{0}^{t} d s \dot{f}^{2},
$$

and we use it with Eqs. (23) and (24) to construct a canonical transformation to coordinates $(\bar{q}, \bar{p})$, obtaining

$$
\bar{q}=q, \bar{p}=(p+m \dot{f})+m \frac{\dot{\gamma}}{\gamma}(q-f),
$$

and

$\bar{H}(\bar{q}, \bar{p}, t)=\frac{\bar{p}^{2}}{2 m}+\frac{1}{\gamma^{2}} U_{0}\left(\frac{\bar{q}-f}{\gamma}\right)-\frac{m}{2} \frac{\ddot{\gamma}}{\gamma}(\bar{q}-f)^{2}-m \ddot{f} \bar{q}$.

The Hamiltonians $H(q, p, t)$ and $\bar{H}(\bar{q}, \bar{p}, t)$ generate equivalent trajectories, in the sense of Eqs. (25) and (26). Moreover, since $q=\bar{q}$, these trajectories are identical in configuration space. This observation can be verified independently by considering the second-order differential equation for the coordinates $q$ and $\bar{q}$. In either case, we have

$$
m \ddot{q}=-\frac{1}{\gamma^{3}} U_{0}{ }^{\prime}[(q-f) / \gamma]+m \frac{\ddot{\gamma}}{\gamma}(q-f)+m \ddot{f} .
$$

Comparing $H$ [Eq. (27)] and $\bar{H}$ [Eq. (30)], we see that the nonlocal terms in the former are replaced by local terms in the latter, which we conceptually identify as a local formulation of $H_{1}(t)$ [Eq. (21)]. The first of these new terms in $\bar{H}$ is an inverted harmonic oscillator whose stiffness is proportional to the acceleration of the scaling factor; cf., also Refs. [16,28]. The quantum analogue of the second term has appeared in the context of linear transport processes. The so-called Duru transformation [38] maps the dynamics of a transport process into the comoving frame $q \rightarrow q-f$, removing the time dependence of the original potential associated with $f(t)$ at the expense of introducing the time dependent force $-m \ddot{f}$, which is precisely cancelled by the counterdiabatic local potential $-m \ddot{f} \bar{q}$.

Now, consider a protocol in which the parameters $f$ and $\gamma$ are fixed outside some interval $t_{0} \leq t \leq t_{1}$ (as in the inset of Fig. 1), and imagine trajectories $z(t)$ and $\bar{z}(t)$ that evolve under $H$ and $\bar{H}$, respectively, from identical initial

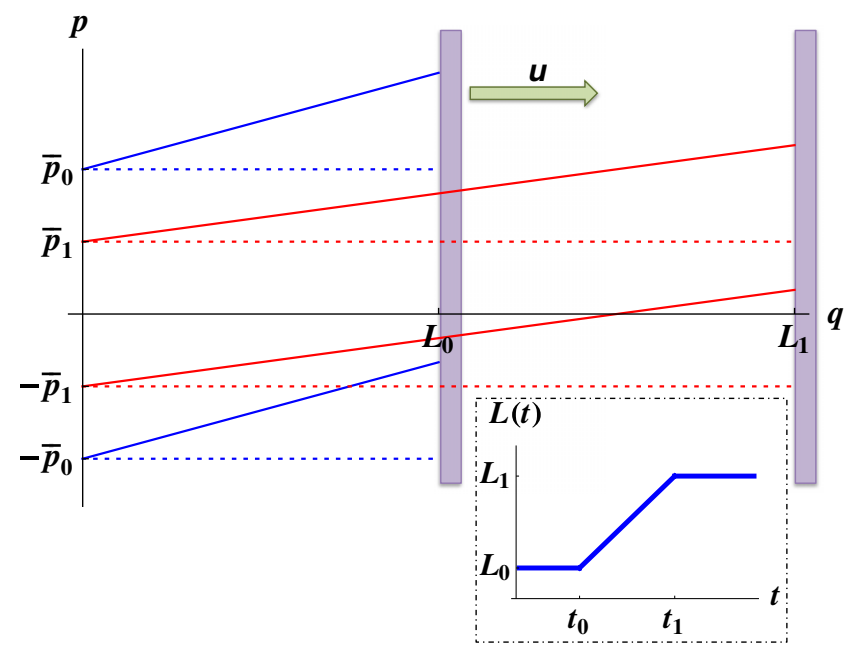

FIG. 1. Shortcut to adiabaticity based on dissipationless classical driving. Energy shells for a particle in a one-dimensional box [Eq. (42)], in a time-dependent piston of width $L(t)$ that changes at a constant rate $u$ for $t_{0} \leq t \leq t_{1}$ (inset). Energy shells corresponding to $H_{0}(\bar{z} ; L)$ are shown as a pair of parallel, dotted line segments of length $L$, at momenta $\pm \bar{p}$. The solid lines represent a level surface of the adiabatic invariant $I$ corresponding to the full, counteradiabatic Hamiltonian $H(\bar{z} ; L)(43)$. At $t=t_{0}$, the force $f(q, t)$ [Eq. (44)] induces a "jump" of trajectories $\bar{z}$ from dashed to solid lines-for $t_{0} \leq t \leq t_{1}$, the adiabatic energy shell is deformed invariantly_and finally, at $t=t_{1}$, force $f(q, t)$ [Eq. (44)] induces jumps back to $\bar{p}$.

conditions at $t<t_{0}$. These equivalent trajectories are related by Eq. (29) at every instant in time, which immediately implies that $z(t)$ and $\bar{z}(t)$ are identical in phase space for $t<t_{0}$; then, their momenta differ during the interval $t_{0} \leq t \leq t_{1}$; and finally, they coincide again at $t=t_{1}$ and remain identical thereafter. Since the adiabatic invariant $\omega$ is preserved exactly along the trajectory $z(t)$, it follows that along the trajectory $\bar{z}(t)$, the initial value of $\omega$ (at $t<t_{0}$ ) is identical to the final values of $\omega$ (at $t>t_{1}$ ), even if it varies at intermediate times. Thus, the local driving Hamiltonian (30) implements a shortcut to adiabatic change of the parameters $f$ and $\gamma$ between some given initial and final values.

To gain further insight, let us construct a new canonical transformation, to variables $(\tilde{q}, \tilde{p})$, using

$$
F(q, \tilde{p}, t)=\frac{1}{\gamma}(q-f) \tilde{p}
$$

Applying Eqs. (23) and (24), we get

$$
\tilde{q}=\frac{q-f}{\gamma}, \quad \tilde{p}=\gamma p
$$




$$
\tilde{H}(\tilde{q}, \tilde{p}, t)=\frac{1}{\gamma^{2}}\left[\frac{\tilde{p}^{2}}{2 m}+U_{0}(\tilde{q})\right]
$$

The transformation (33) is a linear dilation of the coordinate and the reciprocal contraction in momentum space.

The fact that $\tilde{H}(\tilde{q}, \tilde{p}, t)$ is time independent, apart from the factor $1 / \gamma^{2}$, has two interesting consequences. First, the quantity $I(\tilde{q}, \tilde{p})=\gamma^{2} \tilde{H}$ is a dynamical invariant, as follows from direct inspection of Hamilton's equations. If we picture a level surface of $I$ as a closed loop in $\tilde{z}$ space, then under $\tilde{H}$, a trajectory $\tilde{z}(t)$ simply evolves around this loop, at a speed proportional to $1 / \gamma(t)^{2}$. The function $I$ can be expressed in any of the three sets of phase-space coordinates considered above. The resulting functions

$$
\begin{aligned}
I(q, p, t) & =\gamma^{2} \frac{p^{2}}{2 m}+U_{0}\left(\frac{q-f}{\gamma}\right), \\
I(\bar{q}, \bar{p}, t) & =\frac{\gamma^{2}}{2 m}\left[\bar{p}-m \frac{\dot{\gamma}}{\gamma}(\bar{q}-f)-m \dot{f}\right]^{2}+U_{0}\left(\frac{\bar{q}-f}{\gamma}\right) \\
I(\tilde{q}, \tilde{p}) & =\frac{\tilde{p}^{2}}{2 m}+U_{0}(\tilde{q})
\end{aligned}
$$

are all dynamical invariants, along Hamiltonian trajectories generated by $H(z, t), \bar{H}(\bar{z}, t)$, and $\tilde{H}(\tilde{z}, t)$, respectively. This conclusion follows from the equivalence of the trajectories $z(t), \bar{z}(t)$, and $\tilde{z}(t)$, which can also be verified by inspection of Hamilton's equations.

The invariance of $I$ allows us to visualize the evolution of these trajectories, as each one clings to a level surface of $I$ expressed in the given phase-space coordinates. If $f$ is not varied with time, then a level surface of $I(q, p, t)$ gets stretched along $q$ and contracted along $p$ as $\gamma$ increases with time (or the other way around if $\gamma$ decreases); a level surface of $I(\bar{q}, \bar{p}, t)$ additionally acquires a shear along the momentum direction, proportional to $\dot{\gamma}$, as illustrated by the pairs of diagonal lines in Fig. 1. If $f$ is varied with time, then a level surface of $I(q, p, t)$ undergoes translation along the coordinate $q$, and the level surface of $I(\bar{q}, \bar{p}, t)$ additionally undergoes a displacement along $p$ by an amount $m \dot{f}$.

Second, if we introduce the new timelike variable [39]

$$
\tau(t)=\int_{0}^{t} d s \gamma^{-2}(s)
$$

we obtain

$$
\frac{d \tilde{q}}{d \tau}=\frac{\tilde{p}}{m} \quad \text { and } \quad \frac{d \tilde{p}}{d \tau}=-U_{0}^{\prime}(\tilde{q}),
$$

which describe motion under a time-independent Hamiltonian, whose energy shells are the level surfaces of $I(\tilde{q}, \tilde{p})$. Let $[\tilde{q}(\tau), \tilde{p}(\tau)]$ denote a particular solution to these equations of motion. Inverting the canonical transformations in Eqs. (33) and (40), we can immediately use this solution to construct trajectories generated by the Hamiltonians $H$ and $\bar{H}$, namely,

$$
q(t)=\gamma \tilde{q}(\tau), \quad p(t)=\frac{1}{\gamma} \tilde{p}(\tau)
$$

and

$$
\bar{q}(t)=\gamma \tilde{q}(\tau)+f, \quad \bar{p}(t)=\frac{1}{\gamma} \tilde{p}(\tau)+m \dot{\gamma} \tilde{q}(\tau)+m \dot{f}
$$

Hence, trajectories generated by the time-dependent Hamiltonians $H$ and $\bar{H}$ can be constructed directly from trajectories evolving under a time-independent Hamiltonian $\tilde{H}$, which further emphasizes the equivalence between these trajectories. We will exploit these observations in the following discussion of shortcuts for driven multiparticle quantum systems.

Energylike dynamical invariants such as $I$ were intensely studied in the mathematical literature for classical and quantum dynamics. In particular, it can be shown that if (and only if) an energylike invariant exists, then one can find a coordinate transformation, as discussed in the present analysis [29,40-46].

For completeness, we note that the transformation from $(\bar{q}, \bar{p})$ to $(\tilde{q}, \tilde{p})$ is generated by the function

$$
\begin{aligned}
F(\bar{q}, \tilde{p}, t)= & \frac{1}{\gamma}(\bar{q}-f)(\tilde{p}+m \gamma f)+\frac{m}{2} \frac{\dot{\gamma}}{\gamma}(\bar{q}-f)^{2} \\
& +\frac{m}{2} \int_{0}^{t} d s\left(\dot{\gamma}^{2}+2 \ddot{\gamma} \gamma\right),
\end{aligned}
$$

for which we have

$$
\tilde{q}=\frac{1}{\gamma}(\bar{q}-f) \quad \text { and } \quad \tilde{p}=\gamma(\bar{p}-m \dot{f})-m \dot{\gamma}(\bar{q}-f) .
$$

\section{A. An illustrative example: A particle in a time-dependent box}

For a particle in a time-dependent box, the form of the new Hamiltonian $\bar{H}(\bar{q}, \bar{p}, t)$ (30) can be understood intuitively. Consider a particle of mass $m$ inside a onedimensional box with hard walls at $q=0$ and $q=L$, as described by the Hamiltonian

$$
H_{0}(z ; L)=\frac{p^{2}}{2 m}+U_{\mathrm{box}}(q ; L),
$$

where $U_{\mathrm{box}}(q ; L)$ is zero inside the box and "infinite" outside. We further assume that $L=L(t)$ changes with constant rate $u$ for times $t_{0} \leq t \leq t_{1}$ and is constant otherwise with $L\left(t \leq t_{0}\right)=L_{0} \quad$ and $\quad L\left(t \geq t_{1}\right)=L_{1}$; cf. Fig. 1. Now, imagine the aforementioned adiabatic energy shell as a closed loop that is deformed as $L(t)$ is varied with time. Then, $H_{0}(z ; L)$ generates motion around this loop, and the auxiliary CD term $H_{1}(t)=m \dot{L} \xi=m u \xi$ adjusts each trajectory $z$ so that it remains on shell [33]; see 
Fig. 1. The dashed lines represent the adiabatic energy shells corresponding to $H_{0}$ for a particular energy $E$. Notice that particles at $q=L$ hit the hard wall and are "boosted" from one branch to the other. In other words, particles hitting the hard wall with momentum $p$ are reflected at $q=L$ and travel back with $-p$, and so close the loop. The solid lines $p+m u q / L$ and $-p+m u q / L$ represent a level surface of the adiabatic invariant $I$ corresponding to the full counterdiabatic Hamiltonian

$$
H(z ; L)=\frac{p^{2}}{2 m}+U_{\mathrm{box}}(q ; L)+\frac{u}{L} q p .
$$

In the previous discussion, we were asking for a set of coordinates $(\bar{q}, \bar{p})$ and the corresponding Hamiltonian $\bar{H}(\bar{q}, \bar{p}, t)$, for which here the solid lines represent the exact solution. For times $t<t_{0}$ and $t>t_{1}$, the energy shells for old and new coordinates are identical. In other words at $t=t_{0}$ the trajectories $z$ have to "jump" from $p= \pm \sqrt{2 m E}$ to $\bar{p}= \pm p+m u q / L$, where $\bar{q} \equiv q$, and at $t=t_{1}$, the trajectories $z$ jump back to the unperturbed shell. These jumps are induced by a force

$$
\mathfrak{f}(\bar{q}, t)=m \frac{\bar{q} u}{L_{0}} \delta\left(t-t_{0}\right)-m \frac{\bar{q} u}{L_{1}} \delta\left(t-t_{1}\right)
$$

that applies "impulses" at $t=t_{0}$ and $t=t_{1}$. The latter force is the derivative of an auxiliary potential $\mathfrak{f}(\bar{q}, t)=-\partial_{\bar{q}} U_{1}(\bar{q}, t)$

$$
\begin{aligned}
U_{1}(\bar{q}, t) & =-\frac{m}{2} \frac{\bar{q}^{2} u}{L_{0}} \delta\left(t-t_{0}\right)+\frac{m}{2} \frac{\bar{q}^{2} u}{L_{1}} \delta\left(t-t_{1}\right), \\
& =-\frac{m}{2} \frac{\ddot{L}(t)}{L(t)} \bar{q}^{2}
\end{aligned}
$$

that we recognize as the additional potential term in the transformed Hamiltonian $\bar{H}(\bar{q}, \bar{p}, t)$ (30), with $\gamma(t)=$ $L(t) / L(0)$ and $f(t)=0$.

Therefore, we conclude that the additional harmonic term in the Hamiltonian (30) with possible negative spring constant $-m \ddot{\gamma} / \gamma$ is nothing else but the term necessary to facilitate the transfer of the classical trajectories from the energy shells of $H_{0}$ to those invariant under $H$ [Eq. (27)] and eventually $\bar{H}$ [Eq. (30)]. Interestingly enough, this result agrees with the $\mathrm{CD}$ derived in the quantum case for a time-dependent boxlike confinement using Lewis-Riesenfeld invariants and reverse engineering of scaling laws [16].

\section{MULTIPARTICLE QUANTUM SYSTEMS}

In the previous section, we showed how the auxiliary, classical term in the counterdiabatic Hamiltonian can be brought into a local form. We will next apply this finding to general multiparticle quantum systems. Let us consider the broad family of many-body systems described by the Hamiltonian

$$
\hat{\mathcal{H}}_{0}=\sum_{i=1}^{N}\left[-\frac{\hbar^{2}}{2 m} \Delta_{\mathbf{q}_{i}}+U\left[\mathbf{q}_{i}, \lambda(t)\right]\right]+\epsilon(t) \sum_{i<j} V\left(\mathbf{q}_{i}-\mathbf{q}_{j}\right),
$$

with $\mathbf{q}_{i} \in \mathbb{R}^{D}$ unless stated otherwise ( $D$ denoting the effective dimension of the system), and where $\Delta_{\mathbf{q}_{i}}$ is the Laplace operator and $U(\mathbf{q}, t)$ represents an external trap whose time dependence is of the form [Eq. (3)] $U[\mathbf{q}, \lambda(t)]=U_{0}\{[q-\mathbf{f}(t)] / \gamma(t)\} / \gamma(t)^{2}$. As before [Eq. (3)], the trap can be shifted by the time-dependent displacement $\mathbf{f}=\mathbf{f}(t)$ and simultaneously modulated by the scaling factor $\gamma=\gamma(t)$. We further assume that the two-body interaction potential obeys

$$
V(\kappa \mathbf{q})=\kappa^{-\alpha} V(\mathbf{q})
$$

which includes relevant examples in ultracold gases such as the pseudopotential for contact interactions [47], e.g., the Fermi-Huang potential for $s$-wave scattering for which $\alpha=D$ [48].

We define the dimensionless coupling constant $\epsilon(0)=1$ at $t=0$ and consider a stationary state $\Psi(t=0)=\Psi\left(\mathbf{q}_{1}, \ldots, \mathbf{q}_{N} ; t=0\right)$, with chemical potential $\mu$, i.e., $\hat{\mathcal{H}}_{0} \Psi=\mu \Psi$. The scale-invariant solution for this multiparticle quantum system that generalizes the wave function for a single degree of freedom discussed earlier reads

$$
\Psi(t)=\gamma^{-N D / 2} e^{-i \mu \tau / \hbar} \Psi\left[\frac{\mathbf{q}_{1}-\mathbf{f}(t)}{\gamma(t)}, \ldots, \frac{\mathbf{q}_{N}-\mathbf{f}(t)}{\gamma(t)} ; 0\right],
$$

where $\tau$ is the timelike variable introduced above [Eq. (36)]. By substituting the latter ansatz into the many-body Schrödinger equation, we find that $\Psi(t)$ is actually the exact time-dependent solution for the dynamics generated by the CD Hamiltonian

$$
\begin{aligned}
\gamma^{2} \hat{\mathcal{H}}= & \sum_{i=1}^{N}\left[-\frac{\hbar^{2}}{2 m} \Delta_{\tilde{\mathbf{q}}_{i}}+U_{0}\left(\tilde{\mathbf{q}}_{i}\right)\right] \\
& +\epsilon \gamma^{2-\alpha} \sum_{i<j} V\left(\tilde{\mathbf{q}}_{i}-\tilde{\mathbf{q}}_{j}\right) \\
& +\sum_{i=1}^{N}\left[-i \frac{\hbar \partial_{\tau} \mathbf{f}}{\gamma} \cdot \partial_{\tilde{\mathbf{q}}_{i}}-i \frac{\hbar \partial_{\tau} \gamma}{2 \gamma}\left(\tilde{\mathbf{q}}_{i} \cdot \partial_{\tilde{\mathbf{q}}_{i}}+\partial_{\tilde{\mathbf{q}}_{i}} \cdot \tilde{\mathbf{q}}_{i}\right)\right],
\end{aligned}
$$

where the scaled spatial coordinate reads $\tilde{\mathbf{q}}_{i}=$ $\left[\mathbf{q}_{i}-\mathbf{f}(t)\right] / \gamma$, as before. The scale-invariant solution to a related classical and restricted problem with $\mathbf{f}(t)=0$ was derived in Ref. [49]. We observe that in an interacting system (with $V \neq 0$ ), there is an additional consistency condition for the dynamics to be scale invariant:

$$
\epsilon(t)=\gamma(t)^{\alpha-2},
$$


given the definition $\epsilon(0)=1$. Generally, inducing a scaleinvariant dynamics in an interacting system requires us to tune the interaction along the process. In ultracold-atom experiments, this tuning is a routine task in the laboratory assisted by means of a Feshbach resonance [50] or a modulation of the transverse confinement in lowdimensional systems [51,52]. No interaction tuning is required in processes involving transport exclusively, that is, in protocols for which $\mathbf{f}=\mathbf{f}(t)$ and $\gamma(t)=1$. For processes with $\gamma(t) \neq 1$, there are relevant scenarios for which $\alpha=2$ and no interaction tuning is required $[15,16]$. In addition, for processes with $\gamma(t) \neq 1$ and $\alpha \neq 2$, whenever the scaling factor remains of order unity along the process $\gamma(t) \sim \mathcal{O}(1)$, a high-fidelity quantum driving is achieved even in the absence of interaction tuning, i.e., while keeping $\epsilon(t)=1$ [16].

Provided that the consistency equation (50) is fulfilled (or approximately satisfied) so that $\epsilon \gamma^{2-\alpha}=1, \hat{\mathcal{I}}=\gamma^{2} \hat{\mathcal{H}}_{0}$ becomes a first integrand or constant of motion and can be identified as an invariant operator $\hat{\mathcal{I}}$ satisfying

$$
\frac{d \hat{\mathcal{I}}}{d t}=\frac{\partial \hat{\mathcal{I}}}{\partial t}+\frac{1}{i \hbar}[\hat{\mathcal{I}}, \hat{\mathcal{H}}]
$$

and that is the quantum equivalent of the classical, energylike dynamical invariant [Eq. (35)].

The third line in Eq. (49) corresponds to the auxiliary CD Hamiltonian that in the original variables $\left(\mathbf{q}_{i}, \mathbf{p}_{i}\right)$ reads

$$
\hat{\mathcal{H}}_{1}=\sum_{i=1}^{N}\left[\dot{\mathbf{f}} \cdot \mathbf{p}_{i}+\frac{\dot{\gamma}}{2 \gamma}\left\{\mathbf{q}_{i}-\mathbf{f}(t), \mathbf{p}_{i}\right\}\right] .
$$

Here, the curly brackets denote the anticommutator of two operators $A$ and $B:\{A, B\}=A \cdot B+B \cdot A$. In complete analogy to the classical case, the first term is the auxiliary $\mathrm{CD}$ term associated with transport along the trajectory $\mathbf{q}=\mathbf{f}(t)$, while the second one is associated with the expansion. Equation (52) agrees with the singleparticle expression in Eq. (9) and previous results derived for power-law traps $[14,28,33]$.

In the previous section, we found coordinate transformations that allowed us to write the CD Hamiltonian for a system with one degree of freedom in local form. The crucial steps involved finding a generating function for the coordinate transformation and a corresponding dynamical invariant. In the following, we will apply the same ideas to the multiparticle Hamiltonian $\hat{\mathcal{H}}$ (49). The representation in quantum mechanics of the group of linear canonical transformations has been discussed at length in the literature; see, for instance, Ref. [53]. We denote the quantum, multiparticle unitary transformation that plays the role of the classical generating function $F(q, \bar{p}, t)$ [Eq. (28)] by $\mathcal{U}$. It reads

$$
\mathcal{U}=\prod_{i=1}^{N} \exp \left(\frac{i m}{\hbar} \dot{\mathbf{f}} \cdot \mathbf{q}_{i}+\frac{i m \dot{\gamma}}{2 \hbar \gamma}\left(\mathbf{q}_{i}-\mathbf{f}\right)^{2}-i \frac{m}{2} \int_{0}^{t} d s \dot{\mathbf{f}}^{2}\right)
$$

The latter functions transform the "old" set of coordinates $\left(\mathbf{q}_{i}, \mathbf{p}_{i}\right)$ to a new set $\left(\overline{\mathbf{q}}_{i}, \overline{\mathbf{p}}_{i}\right)$ according to

$$
\begin{gathered}
\mathbf{q}_{i} \rightarrow \overline{\mathbf{q}}_{i}=\mathcal{U} \mathbf{q}_{i} \mathcal{U}^{\dagger}=\mathbf{q}_{i}, \\
\mathbf{p}_{i} \rightarrow \overline{\mathbf{p}}_{i}=\mathcal{U} \mathbf{p}_{i} \mathcal{U}^{\dagger}=\mathbf{p}_{i}-\frac{m \dot{\gamma}}{\gamma}\left(\mathbf{q}_{i}-\mathbf{f}\right)-m \dot{\mathbf{f}}, \\
\hat{\mathcal{H}} \rightarrow \hat{\overline{\mathcal{H}}}(t)=\mathcal{U} \hat{\mathcal{H}}(t) \mathcal{U}^{\dagger}-i \hbar \mathcal{U} \partial_{t} \mathcal{U}^{\dagger} .
\end{gathered}
$$

Here, the new representation of the CD Hamiltonian becomes

$$
\begin{aligned}
\hat{\mathcal{H}}(t)= & \sum_{i=1}^{N}\left[-\frac{\hbar^{2}}{2 m} \Delta_{\overline{\mathbf{q}}_{i}}+U\left[\overline{\mathbf{q}}_{i}, \lambda(t)\right]\right]+\epsilon(t) \sum_{i<j} V\left(\overline{\mathbf{q}}_{i}-\overline{\mathbf{q}}_{j}\right) \\
& +\sum_{i=1}^{N}\left[-\frac{m}{2} \frac{\ddot{\gamma}}{\gamma}\left(\overline{\mathbf{q}}_{i}-\mathbf{f}\right)^{2}-m \ddot{\mathbf{f}} \cdot \overline{\mathbf{q}}_{i}\right]
\end{aligned}
$$

which is the multiparticle quantum equivalent of the classical Hamiltonian $\bar{H}(\bar{q}, \bar{p}, t)$ (30). Under this canonical transformation, the time evolution of the initial state is mapped to $\Psi(t) \rightarrow \Phi(t)=\mathcal{U} \Psi(t)$. Finally, it follows that the dynamical invariant $\mathcal{I}$ can be written in new coordinates $\left(\overline{\mathbf{q}}_{i}, \overline{\mathbf{p}}_{i}\right)$

$$
\begin{aligned}
\hat{\mathcal{I}}= & \sum_{i=1}^{N} \frac{1}{2 m}\left[\gamma\left(\overline{\mathbf{p}}_{i}-m \dot{f}\right)-m \dot{\gamma}\left(\overline{\mathbf{q}}_{i}-\mathbf{f}\right)\right]^{2} \\
& +\sum_{i=1}^{N} U_{0}\left(\frac{\overline{\mathbf{q}}_{i}-\mathbf{f}}{\gamma}\right)+\sum_{i<j} V\left(\frac{\overline{\mathbf{q}}_{i}}{\gamma}-\frac{\overline{\mathbf{q}}_{j}}{\gamma}\right),
\end{aligned}
$$

which is equivalent to the second line of Eq. (35).

We note that the dynamics governed by $\mathcal{H}(t)$ induces a phase modulation associated with $\mathcal{U}$ that generally leads to excitations away from the adiabatic trajectory $\Psi(t)$. The nonadiabatic nature of the resulting shortcuts to adiabaticity is exclusively captured by $\mathcal{U}$ (phase modulations), while local correlation functions are identical at all times with the adiabatic ones, given that $|\Psi(t)|^{2}=|\Phi(t)|^{2}$. Nonetheless, $\mathcal{U}$ reduces to the identity and $\Phi(t)=\Psi(t)$ at the beginning and end of the process (e.g., at time $t=\left\{0, \tau_{F}\right\}$ ). Further, it is straightforward to design protocols involving only smooth modulations of the auxiliary counterdiabatic field (requiring no impulses) of relevance to experimental realizations, as we shall discuss in Sec. VIII. In the following sections, the formalism just described will prove useful to engineer shortcuts to adiabaticity for several nontrivial systems. 


\section{LOCAL COUNTERDIABATIC DRIVING FOR AN ARBITRARY TRAPPING POTENTIAL}

The only condition we have imposed in the preceding sections on the time dependence of the external potential is its scale-invariant form [Eq. (3)]. To illustrate the generality of our approach, let $U(\mathbf{q}, t)$ have a power-series expansion

$$
U(\mathbf{q}, t)=\sum_{p=0}^{\infty} \alpha_{p}(t)(\mathbf{q}-\mathbf{f})^{p},
$$

TABLE I. List of counterdiabatic driving schemes for potentials that acquire the form of $U(q, t)$ in Eq. (3) under the indicated time dependence of the parameters $A, B$, and $\alpha$, including well-known shape-invariant potentials in supersymmetric quantum mechanics. A local CD protocol for expansions and compressions $(\mathbf{f}=0)$ is induced by the potential $\bar{U}(\bar{q}, t)=U(\bar{q}, t)-m \ddot{\gamma} \bar{q}^{2} /(2 \gamma)$. When meaningful, the same potentials can be used as a two-body potential $V$ with $\alpha=2$. A single degree of freedom is considered for clarity; the extension to higher dimensions under cylindrical or spherical symmetry is straightforward following Ref. [16]. The range of potentials is $-\infty \leq q \leq \infty, 0 \leq r \leq \infty$, unless stated otherwise. The family of power-law potentials [28,33] includes the harmonic case $(b=2)[15]$ and the infinite square well $(b=\infty)[16]$.

\begin{tabular}{|c|c|c|c|}
\hline Name & $U(q, t)$ & Time dependence & Counterdiabatic modulation $-\ddot{\gamma} / \gamma$ \\
\hline Arbitrary potential & $\frac{1}{\gamma^{2}} U_{0}\left(\frac{q}{\gamma}\right)$ & Arbitrary $\gamma(t)$ & $-\frac{\ddot{\gamma}(t)}{\gamma(t)}$ \\
\hline Power-law trap & $A|q|^{b}$ & $A(t)=\frac{A(0)}{\gamma^{2+b}}$ & $\frac{\dot{A}(t)}{(2+b) A(t)}-\frac{3+b}{(2+b)^{2}}\left[\frac{\dot{A}(t)}{A(t)}\right]^{2}$ \\
\hline $\begin{array}{l}\text { Modified Pöschl-Teller } \\
\text { well [55] }(\lambda>1)\end{array}$ & $-\frac{\hbar^{2}}{2 m} \alpha^{2} \frac{\lambda(\lambda-1)}{\cosh ^{2} \alpha q}$ & $\alpha(t)=\frac{\alpha(0)}{\gamma}$ & $\frac{\ddot{\alpha}(t)}{\alpha(t)}-2\left[\frac{\dot{\alpha}(t)}{\alpha(t)}\right]^{2}$ \\
\hline $\begin{array}{l}\text { Pöschl-Teller well [55] } \\
(\lambda, \kappa>1)\end{array}$ & $\frac{\hbar^{2}}{2 m} \alpha^{2}\left(\frac{\lambda(\lambda-1)}{\cos ^{2} \alpha q}+\frac{\kappa(\kappa-1)}{\sin ^{2} \alpha q}\right)$ & $\alpha(t)=\frac{\alpha(0)}{\gamma}$ & $\frac{\ddot{\alpha}(t)}{\alpha(t)}-2\left[\frac{\dot{\alpha}(t)}{\alpha(t)}\right]^{2}$ \\
\hline Optical lattice & $A \sin ^{2}(\alpha q)$ & $A(t)=\frac{A(0)}{\gamma^{2}}, \alpha(t)=\frac{\alpha(0)}{\gamma}$ & $\frac{\dot{A}(t)}{2 A(t)}-\frac{3}{4}\left[\frac{\dot{A}(t)}{A(t)}\right]^{2}=\frac{\ddot{\alpha}(t)}{\alpha(t)}-2\left[\frac{\dot{\alpha}(t)}{\alpha(t)}\right]^{2}$ \\
\hline Gaussian well & $-A \exp \left(-\alpha^{2} q^{2}\right)$ & $A(t)=\frac{A(0)}{\gamma^{2}}, \alpha(t)=\frac{\alpha(0)}{\gamma}$ & $\frac{\dot{A}(t)}{2 A(t)}-\frac{3}{4}\left[\frac{\dot{A}(t)}{A(t)}\right]^{2}=\frac{\ddot{\alpha}(t)}{\alpha(t)}-2\left[\frac{\dot{\alpha}(t)}{\alpha(t)}\right]^{2}$ \\
\hline Finite square well & $-A \Theta(\alpha-q)$ & $A(t)=\frac{A(0)}{\gamma^{2}}, \alpha(t)=\alpha(0) \gamma$ & $\frac{\dot{A}(t)}{2 A(t)}-\frac{3}{4}\left[\frac{\dot{A}(t)}{A(t)}\right]^{2}=-\frac{\ddot{\alpha}(t)}{\alpha(t)}$ \\
\hline Exponential & $-A \exp (-r / \alpha)$ & $A(t)=\frac{A(0)}{\gamma^{2}}, \alpha(t)=\alpha(0) \gamma$ & $\frac{\dot{A}(t)}{2 A(t)}-\frac{3}{4}\left[\frac{\dot{A}(t)}{A(t)}\right]^{2}=-\frac{\ddot{\alpha}(t)}{\alpha(t)}$ \\
\hline Yukawa & $-A \frac{\exp (-r / \alpha)}{r / \alpha}$ & $A(t)=\frac{A(0)}{\gamma^{2}}, \alpha(t)=\alpha(0) \gamma$ & $\frac{\dot{A}(t)}{2 A(t)}-\frac{3}{4}\left[\frac{\dot{\dot{A}(t)}}{\dot{A}(t)}\right]^{2}=-\frac{\ddot{\alpha}(t)}{\alpha(t)}$ \\
\hline $\begin{array}{l}\text { Wood-Saxon } \\
(\alpha \ll f)\end{array}$ & $-A \frac{\exp (-r / \alpha)}{1-\exp (r / \alpha)}$ & $A(t)=\frac{A(0)}{\gamma^{2}}, \alpha(t)=\alpha(0) \gamma$ & $\frac{\dot{A}(t)}{2 A(t)}-\frac{3}{4}\left[\frac{\dot{A}(t)}{A(t)}\right]^{2}=-\frac{\ddot{\alpha}(t)}{\alpha(t)}$ \\
\hline $\begin{array}{l}\text { Hulthén } \\
(\alpha \ll f)\end{array}$ & $-\frac{A}{1+\exp [(r-f) / \alpha]}$ & $A(t)=\frac{A(0)}{\gamma^{2}}, \alpha(t)=\alpha(0) \gamma$ & $\frac{\dot{A}(t)}{2 A(t)}-\frac{3}{4}\left[\frac{\dot{A}(t)}{A(t)}\right]^{2}=-\frac{\ddot{\alpha}(t)}{\alpha(t)}$ \\
\hline Kratzer & $-2 A\left(\frac{\alpha}{r}-\frac{1}{2} \frac{\alpha^{2}}{r^{2}}\right)$ & $A(t)=\frac{A(0)}{\gamma^{2}}, \alpha(t)=\alpha(0) \gamma$ & $\frac{\dot{A}(t)}{2 A(t)}-\frac{3}{4}\left[\frac{\dot{A}(t)}{A(t)}\right]^{2}=-\frac{\ddot{\alpha}(t)}{\alpha(t)}$ \\
\hline Morse & $\begin{array}{c}A^{2}+B^{2} \exp (-2 \alpha q) \\
-2 B(A+\alpha / 2) \exp (-\alpha q)\end{array}$ & $A(t)=\frac{A(0)}{\gamma}, B(t)=\frac{B(0)}{\gamma}, \alpha(t)=\frac{\alpha(0)}{\gamma}$ & $\begin{array}{l}\frac{\ddot{X}(t)}{X(t)}-2\left[\frac{\dot{X}(t)}{X(t)}\right]^{2} \\
(X=A, B, \alpha)\end{array}$ \\
\hline $\begin{array}{l}\text { Eckart } \\
\left(B>A^{2}\right)\end{array}$ & $\begin{array}{c}A^{2}+B^{2} / A^{2}-2 B \operatorname{coth} \alpha q \\
+A(A-\alpha) \operatorname{cosech}^{2} \alpha q\end{array}$ & $A(t)=\frac{A(0)}{\gamma}, B(t)=\frac{B(0)}{\gamma^{2}}, \alpha(t)=\frac{\alpha(0)}{\gamma}$ & $\begin{aligned} \frac{\ddot{X}(t)}{X(t)}-2\left[\frac{\dot{X}(t)}{X(t)}\right]^{2} & =\frac{\dot{B}(t)}{2 B(t)}-\frac{3}{4}\left[\frac{\dot{B}(t)}{B(t)}\right]^{2} \\
(X & =A, \alpha)\end{aligned}$ \\
\hline $\begin{array}{l}\text { Scarf I } \\
\text { (Trigonometric) }\end{array}$ & $\begin{array}{c}-A^{2}+\left(A^{2}+B^{2}-A \alpha\right) \sec ^{2} \alpha q \\
-B(2 A-\alpha) \tan \alpha q \sec \alpha q\end{array}$ & $A(t)=\frac{A(0)}{\gamma}, B(t)=\frac{B(0)}{\gamma}, \alpha(t)=\frac{\alpha(0)}{\gamma}$ & $\begin{array}{l}\frac{\ddot{X}(t)}{X(t)}-2\left[\frac{\dot{X}(t)}{X(t)}\right]^{2} \\
(X=A, B, \alpha)\end{array}$ \\
\hline $\begin{array}{l}\text { Scarf II } \\
\text { (Hyperbolic) }\end{array}$ & $\begin{array}{c}A^{2}+\left(B^{2}-A^{2}-A \alpha\right) \operatorname{sech}^{2} \alpha q \\
+B(2 A+\alpha) \operatorname{sech} \alpha q \tanh \alpha q\end{array}$ & $A(t)=\frac{A(0)}{\gamma}, B(t)=\frac{B(0)}{\gamma}, \alpha(t)=\frac{\alpha(0)}{\gamma}$ & $\begin{array}{l}\frac{\ddot{X}(t)}{X(t)}-2\left[\frac{\dot{X}(t)}{X(t)}\right]^{2} \\
(X=A, B, \alpha)\end{array}$ \\
\hline $\begin{array}{l}\text { Generalized Pöschl-Teller } \\
(A<B)\end{array}$ & $\begin{array}{l}A^{2}+\left(B^{2}+A^{2}+A \alpha\right) \operatorname{cosech}^{2} \alpha r \\
-B(2 A+\alpha) \operatorname{coth} \alpha r \operatorname{cosech} \alpha r\end{array}$ & $A(t)=\frac{A(0)}{\gamma}, B(t)=\frac{B(0)}{\gamma}, \alpha(t)=\frac{\alpha(0)}{\gamma}$ & $\begin{array}{l}\frac{\ddot{X}(t)}{X(t)}-2\left[\frac{\dot{X}(t)}{X(t)}\right]^{2} \\
(X=A, B, \alpha)\end{array}$ \\
\hline $\begin{array}{l}\text { Pöschl-Teller II } \\
(B<A)\end{array}$ & $\begin{array}{c}(A-B)^{2}-A(A+\alpha) \operatorname{sech}^{2} \alpha r \\
+B(B-\alpha) \operatorname{cosech} \alpha r\end{array}$ & $A(t)=\frac{A(0)}{\gamma}, B(t)=\frac{B(0)}{\gamma}, \alpha(t)=\frac{\alpha(0)}{\gamma}$ & $\begin{array}{c}\frac{\ddot{X}(t)}{X(t)}-2\left[\frac{\dot{X}(t)}{X(t)}\right]^{2} \\
(X=A, B, \alpha)\end{array}$ \\
\hline $\begin{array}{l}\text { Rosen-Morse I } \\
\text { (Trigonometric) }\end{array}$ & $\begin{array}{c}A(A-\alpha) \operatorname{cosec}^{2} \alpha q+2 B \cot \alpha q \\
-A^{2}+B^{2} / A^{2}\end{array}$ & $A(t)=\frac{A(0)}{\gamma}, B(t)=\frac{B(0)}{\gamma^{2}}, \alpha(t)=\frac{\alpha(0)}{\gamma}$ & $\begin{array}{c}\frac{\ddot{X}(t)}{X(t)}-2\left[\frac{\dot{X}(t)}{X(t)}\right]^{2}=\frac{\dot{B}(t)}{2 B(t)}-\frac{3}{4}\left[\frac{\dot{B}(t)}{B(t)}\right]^{2} \\
(X=A, \alpha)\end{array}$ \\
\hline$(0 \leq \alpha q \leq \pi)$ & & & \\
\hline $\begin{array}{l}\text { Rosen-Morse II } \\
\text { (Hyperbolic) }\left(B<A^{2}\right)\end{array}$ & $\begin{array}{c}A^{2}+B^{2} / A^{2}-A(A+\alpha) \operatorname{sech}^{2} \alpha q \\
+2 B \tanh \alpha q\end{array}$ & $A(t)=\frac{A(0)}{\gamma}, B(t)=\frac{B(0)}{\gamma^{2}}, \alpha(t)=\frac{\alpha(0)}{\gamma}$ & $\begin{array}{c}\frac{\ddot{X}(t)}{X(t)}-2\left[\frac{\dot{X}(t)}{X(t)}\right]^{2}=\frac{\dot{B}(t)}{2 B(t)}-\frac{3}{4}\left[\frac{\dot{B}(t)}{B(t)}\right]^{2} \\
(X=A, \alpha)\end{array}$ \\
\hline
\end{tabular}


where $\alpha_{p}(t)=U_{0}^{(p)}(\mathbf{0}) / p$ !, with $U_{0}^{(p)}$ denoting the $p$ th derivative and $U_{0}^{(0)}(\mathbf{0}, t)=U_{0}(\mathbf{0}, t)$. Further, assume the potential to be isotropic, the extension to anisotropic potentials being straightforward. Given a process governed by the time-dependent potential [Eq. (57)], we aim at finding a local CD protocol.

First, let us impose the form required for scaling laws $U(\mathbf{q}, t)=U_{0}[(\mathbf{q}-\mathbf{f}) / \gamma] / \gamma^{2}$ [Eq. (3)], which implies the following relationship among the coefficients in the series expansion [Eq. (57)]:

$$
\alpha_{0}(t)=\frac{\alpha_{0}(0)}{\gamma^{2}}, \quad \alpha_{1}(t)=\frac{\alpha_{1}(0)}{\gamma^{3}}, \ldots, \quad \alpha_{p}(t)=\frac{\alpha_{p}(0)}{\gamma^{p+2}} .
$$

Hence, for an arbitrary potential, the time modulation in Eq. (57) can be implemented, provided that each coefficient $\alpha_{p}(t)$ can be tuned independently. The latter condition leads to the recurrence relation

$\frac{\alpha_{p}(t)}{\alpha_{p}(0)}=\left[\frac{\alpha_{p-1}(t)}{\alpha_{p-1}(0)}\right]^{(p+2) /(p+1)}=\left[\frac{\alpha_{p-m}(t)}{\alpha_{p-m}(0)}\right]^{(p+2) /(p-m+2)}$,

where the last exponent results from $\prod_{k=1}^{m}(p-k+3) /$ $(p-k+2)=(p+2) /(p-m+2)$. The auxiliary potential terms in Eq. (55) can be absorbed into the definition of the expansion coefficients

$$
\tilde{\alpha}_{p}(t)=\alpha_{p}(t)-\frac{m \ddot{\gamma}}{2 \gamma} \delta_{p, 2}-m \ddot{f}\left(\delta_{p, 1}+\delta_{p, 0}\right),
$$

so that the local CD potential is given by $\bar{U}(\overline{\mathbf{q}}, t)=$ $\sum_{p=0}^{\infty} \tilde{\alpha}_{p}(t)(\overline{\mathbf{q}}-\mathbf{f})^{p}$, which is the sum of the one-body trapping potential $U(\mathbf{q}, t)$ and the auxiliary terms in $\hat{\mathcal{H}}(t)$. The required modulation of the $\tilde{\alpha}_{p}(t)$ coefficients makes the implementation of $\mathrm{CD}$ protocols with nonharmonic traps particularly amenable to the painting-potential technique [54].

\section{A. Example: The quartic trap potential}

As an illustrative example, we consider the quartic potential

$$
U(\mathbf{q}, t)=\alpha_{2}(t)(\mathbf{q}-\mathbf{f})^{2}+\alpha_{4}(t)(\mathbf{q}-\mathbf{f})^{4},
$$

where the time modulations

$$
\alpha_{2}(t)=\frac{\alpha_{2}(0)}{\gamma^{4}} \quad \text { and } \quad \alpha_{4}(t)=\frac{\alpha_{4}(0)}{\gamma^{6}}
$$

lead to a scaling of the form [Eq. (57)], associated with a scale-invariant dynamics. Provided that Eq. (50) is satisfied, the CD potential, for which $\Phi(t)=\mathcal{U} \Psi(t)$ is the exact solution to the many-body Schrödinger equation, is simply given by

$$
\begin{aligned}
\bar{U}(\overline{\mathbf{q}}, t)= & -m \ddot{\mathbf{f}} \cdot \overline{\mathbf{q}}+\left(\frac{\alpha_{2}(0)}{\gamma^{4}}-\frac{m \ddot{\gamma}}{2 \gamma}\right)(\overline{\mathbf{q}}-\mathbf{f})^{2} \\
& +\frac{\alpha_{4}(0)}{\gamma^{6}}(\overline{\mathbf{q}}-\mathbf{f})^{4} .
\end{aligned}
$$

In many instances, the coefficients in the set $\left\{\alpha_{p}(0)\right\}_{p}$ associated with the power series [Eq. (57)] are interrelated, and the required time dependence of the potential can be brought into the form of Eq. (35) by direct inspection or a scaling analysis. A list of examples and the associated CD protocols is provided in Table I, which includes among others the family of shape-invariant potentials in supersymmetric quantum mechanics [56], as well as common potentials in atom optics, such as several types of wells and optical lattices.

\section{COUNTERDIABATIC DRIVING OF NONLINEAR SYSTEMS}

The original formulation of $\mathrm{CD}$ is restricted to linear systems. However, it was recently shown that it can be generalized to nonlinear systems undergoing scaleinvariant expansions and compressions [28]. The approach developed in the previous sections also allows us to treat nonlinear systems.

Typically, nonlinear ("quantum") systems are described by effective evolution equations derived within a meanfield approach. A prominent example is the description of Bose-Einstein condensates, where scale-invariant dynamics is of great relevance to time-of-flight measurements [57-59]. The dynamics is described by the time-dependent Gross-Pitaevskii equation (TDGPE) governing the (normalized) wave function $\Psi(\mathbf{q}, t)$ of a Bose-Einstein condensate

$i \hbar \partial_{t} \Psi(\mathbf{q}, t)=\left[-\frac{\hbar^{2}}{2 m} \Delta_{\mathbf{q}}+U(\mathbf{q}, t)+g_{D}|\Psi(\mathbf{q}, t)|^{2}\right] \Psi(\mathbf{q}, t)$,

where $U(\mathbf{q}, t)$ shall again be of the scale-invariant form [Eq. (3)]. This nonlinear Schrödinger equation can be obtained from the model describing a $D$-dimensional many-body Bose gas with regularized contact interactions (the Fermi-Huang pseudopotential) using a mean-field approximation (e.g., assuming that the many-body wave function takes the form of a Hartree product). We can then expect that a protocol derived in the previous section, exact for the many-body description, should carry over the TDGPE. Indeed, a stationary state $\Psi$ at $t=0$ with chemical potential $\mu$ can be forced to obey a scale-invariant ansatz of the form $\Psi(\mathbf{q}, t)=$ $\exp [-i \mu \tau(t) / \hbar] \gamma^{-D / 2} \Psi[(\mathbf{q}-\mathbf{f}) / \gamma, t=0]$. This ansatz is 
the exact solution to a counterdiabatic nonlinear evolution equation, the modified nonlocal TDGPE

$$
\begin{aligned}
i \hbar \partial_{t} \Psi(\mathbf{q}, t)= & {\left[-\frac{\hbar^{2}}{2 m} \Delta_{\mathbf{q}}+U(\mathbf{q}, t)+\dot{\mathbf{f}} \cdot \mathbf{p}\right.} \\
& \left.+\frac{\dot{\gamma}}{2 \gamma}\{\mathbf{q}-\mathbf{f}, \mathbf{p}\}+g_{D}|\Psi(\mathbf{q}, t)|^{2}\right] \Psi(\mathbf{q}, t),
\end{aligned}
$$

provided that the interaction coupling strength is tuned according to $g_{D}=g_{D}(t=0) \gamma^{D-2}$. We observe that this time dependence of the nonlinear interactions agrees with that required in the $D$-dimensional Bose gas and can be achieved, for instance, by tuning a magnetic field through a Feshbach resonance [50]. Alternatively, in lowdimensional quantum gases $(D=1,2)$, it can be implemented by modulating the transverse confinement $[51,52]$.

As before, the modified TDGPE can be brought into local form by applying the canonical time-dependent transformation $\mathcal{U}$ [Eq. (53)] $(N=1)$, which leads to

$$
\begin{aligned}
i \hbar \partial_{t} \Phi(\overline{\mathbf{q}}, t)= & {\left[-\frac{\hbar^{2}}{2 m} \Delta_{\overline{\mathbf{q}}}+U(\overline{\mathbf{q}}, t)-\frac{m}{2} \frac{\ddot{\gamma}}{\gamma}(\overline{\mathbf{q}}-\mathbf{f})^{2}\right.} \\
& \left.-m \ddot{\mathbf{f}} \cdot \overline{\mathbf{q}}+g_{D}|\Phi(\overline{\mathbf{q}}, t)|^{2}\right] \Phi(\overline{\mathbf{q}}, t),
\end{aligned}
$$

with exclusively local potential terms, and $\Phi=\mathcal{U} \Psi$.

Kundu [60] has shown that inhomogeneous nonlinear Schrödinger equations of this class are equivalent to the standard homogeneous nonlinear Schrödinger equation and hence admit a zero-curvature representation, explaining their integrability [61].

In addition, $\mathrm{CD}$ can also be applied to other examples of nonlinear evolution, for which a nonzero-curvature representation has not been found to date. A relevant instance is the mean-field theory developed by Kolomeisky et al. $[62,63]$ that accurately describes the ground-state density profile of one-dimensional bosons with hard-core contact interactions, i.e., a Tonks-Girardeau gas [64] (and its dual system under Bose-Fermi duality, a one-dimensional spinpolarized Fermi gas [65]), up to spatial antibunching [66]. The time-dependent version of the Kolomeisky equation reads

$$
\begin{aligned}
i \hbar \partial_{t} \Psi(q, t)= & {\left[-\frac{\hbar^{2}}{2 m} \partial_{q}^{2}+U(q, t)\right.} \\
& \left.+\frac{\pi^{2} \hbar^{2}}{2 m}|\Psi(q, t)|^{4}\right] \Psi(q, t),
\end{aligned}
$$

where $\Psi(q, 0)=\sqrt{n(q, 0)}$ is the square root of the initial density profile. CD protocols can be directly obtained from the one-dimensional version of Eqs. (65) and (66), respectively, by replacing $g_{1}|\Psi(\mathbf{q}, t)|^{2}$ by $\pi^{2} \hbar^{2} / 2 m|\Psi(q, t)|^{4}$. (Recall that $|\Phi(q, t)|=|\Psi(q, t)|$.) Note that the quintic nonlinearity arises from the repulsive contact interactions and can be considered as a potential term with $\alpha=2$. As a result, no interaction tuning is required, provided that the gas remains in the Tonks-Girardeau regime. It is worth emphasizing that, as a mean-field theory, the model by Kolomeisky et al. [Eq. (67)] overestimates phase coherence. Therefore, while it properly describes the scaleinvariant dynamics in a time-dependent harmonic trap [67], it fails to accurately account for processes involving interference such as splitting and recombination [65]. Nonetheless, the Kolomeisky equation has been successfully applied to describe the formation of shock waves and it is accurate as long as changes in the density occur on a length scale larger than the "Fermi" length [68]. As a result, under scale-invariant driving, protocols derived from Eq. (67) agree with those designed using an exact many-body treatment $[15,16]$.

We close this section by mentioning that other nonlinear processes that can be assisted by CD include the (meanfield) growth dynamics of a Bose-Einstein condensate [69]. Nonetheless, phase fluctuations in the newborn condensate are expected to result in the formation of solitons [70] or vortices [71], depending in the dimensionality, as dictated by the Kibble-Zurek mechanism [72,73].

\section{COUNTERDIABATIC DRIVING AND REVERSE ENGINEERING: SCALING LAWS}

In the previous discussion, we showed that $\mathrm{CD}$ can be used to enforce scale-invariant dynamics in which the scaling factor follows the adiabatic trajectory. However, there are more general scaling laws that are associated with an invariant of motion, provided that a set of consistency equations is satisfied [74]. Knowledge of these scaling laws paves the way to engineering shortcuts to adiabatic scaleinvariant processes. For a large family of many-particle systems in a time-dependent harmonic trap, such a design was reported in Ref. [15], extending previous results for the single-particle Schrödinger equation [13] as well as the Gross-Pitaevskii equation describing Bose-Einstein condensates in the mean field [75]. These results have been further extended to time-dependent boxlike confinements [16] and arbitrary power-law potentials [28,33]. We next present the general scaling laws associated with a family of Hamiltonians that include all the aforementioned results. Having done so, we shall establish their explicit relation with CD. Let us consider the Hamiltonian (46) in which harmonic and linear terms in $\mathbf{q}_{i}$ are explicitly written as

$$
\begin{aligned}
\hat{\mathcal{H}}_{0}= & \sum_{i=1}^{N}\left[-\frac{\hbar^{2}}{2 m} \Delta_{\mathbf{q}_{i}}+U\left(\mathbf{q}_{i}, t\right)\right]+\epsilon(t) \sum_{i<j} V\left(\mathbf{q}_{i}-\mathbf{q}_{j}\right) \\
& +\sum_{i=1}^{N}\left[\frac{m}{2} \omega^{2}(t)\left[\mathbf{q}_{i}-\mathbf{f}(t)\right]^{2}+m \mathbf{F}(t) \cdot \mathbf{q}_{i}\right],
\end{aligned}
$$


with an arbitrary modulation of the coefficients $\omega(t)$ (harmonic trap frequency) and $\mathbf{F}(t)$. A stationary state $\Psi$ of the system [Eq. (68)] at $t=0$ follows a scale-invariant evolution

$$
\begin{aligned}
& \Phi\left(\left\{\mathbf{q}_{i}\right\}, t\right) \\
& \quad=e^{-(i / \hbar) \int_{0}^{t} d t^{\prime}(m / 2) \dot{\mathbf{f}}^{2}} e^{i \sum_{i=1}^{N}(m \dot{\gamma} / 2 \gamma \hbar)\left(\mathbf{q}_{i}-\mathbf{f}\right)^{2}+(i m / \hbar) \dot{\mathbf{f}} \cdot \mathbf{q}_{i}} \Psi(t),
\end{aligned}
$$

with $\Psi(t)$ given by Eq. (48), whenever the following consistency conditions are satisfied:

$$
\omega^{2}(t)=\frac{\omega_{0}^{2}}{\gamma^{4}}-\frac{\ddot{\gamma}}{\gamma}, \quad \mathbf{F}(t)=-\ddot{\mathbf{f}}, \quad \epsilon(t)=\gamma^{\alpha-2},
$$

with $\omega_{0}=\omega(0)$, and satisfying $\gamma=\gamma(t)$ the boundary conditions $\gamma(0)=1$ and $\dot{\gamma}(0)=0$.

Generally, the resulting dynamics are not adiabatic. Only in the adiabatic limit, where $\ddot{\gamma} \rightarrow 0$ and $\ddot{\mathbf{f}} \rightarrow 0$ in Eq. (70), do we find that the solution for the scaling factor takes the form $\omega^{2}(t)=\omega_{0}^{2} / \gamma^{4}$ and $\mathbf{F}(t)=0$. Nonetheless, counterdiabatic driving provides means to induce finite-time evolution [Eq. (69)] that effectively follows the adiabatic trajectory of the scaling factor. The frequency of the trap is to be replaced by $[15,28]$

$$
\omega^{2}(t) \rightarrow \omega^{2}(t)-\frac{\ddot{\gamma}}{\gamma}=\omega^{2}(t)-\frac{3}{4} \frac{\dot{\omega}^{2}}{\omega^{2}}+\frac{1}{2} \frac{\ddot{\omega}}{\omega},
$$

while the modulation of the linear term remains $\mathbf{F}(t) \rightarrow-\ddot{\mathbf{f}}$.

\section{ENGINEERING SHORTCUTS TO ADIABATICITY ASSISTED BY SMOOTH COUNTERDIABATIC FIELDS}

In the last part of the present analysis, we shall illustrate how the time dependence of the control parameters is to be designed to engineer shortcuts to adiabaticity based on $\mathrm{CD}$, without the requirement of impulse auxiliary fields. Assume we wish to find a shortcut to an adiabatic expansion or compression by changing the scaling factor $\gamma$ from an initial value $\gamma(t=0)=1$ to a final value $\gamma_{F}$ at $t=\tau_{F}$, while at the same time transporting the system by shifting the external trapping potential from $\mathbf{f}(t=0)=0$ to $\mathbf{f}\left(\tau_{F}\right)=\mathbf{f}_{F}$. Further, let us impose that the auxiliary Hamiltonian is switched on at $t=0$ and switched off at $t=\tau_{F}$; cf., the example in Sec. III, i.e., $\hat{\mathcal{H}}=\hat{\mathcal{H}}_{0}$ at $t=\left\{0, \tau_{F}\right\}$. We have seen earlier that the auxiliary nonlocal term $\hat{\mathcal{H}}_{1}$ [Eq. (52)] induces the adiabatic dynamics along the instantaneous eigenstates of the system Hamiltonian $\hat{\mathcal{H}}_{0}$ [Eq. (46)]. The time-dependent coefficient of Eq. (52) is governed by the rate of change of the scaling factor $\gamma$ and the shift function $\mathbf{f}$. Therefore, for it to vanish at $t=\left\{0, \tau_{F}\right\}$, the following boundary conditions are required:

$$
\gamma(0)=1, \quad \dot{\gamma}(0)=0, \quad \gamma\left(\tau_{F}\right)=\gamma_{F}, \quad \dot{\gamma}\left(\tau_{F}\right)=0
$$

and

$$
\mathbf{f}(0)=\mathbf{0}, \quad \dot{\mathbf{f}}(0)=\mathbf{0}, \quad \mathbf{f}\left(\tau_{F}\right)=\mathbf{f}_{F}, \quad \dot{\mathbf{f}}\left(\tau_{F}\right)=\mathbf{0},
$$

which can be used to determine an interpolating ansatz, for instance,

$$
\begin{aligned}
& \gamma(t)=1+3\left(\gamma_{F}-1\right) \frac{t^{2}}{\tau_{F}^{2}}+2\left(\gamma_{F}-1\right) \frac{t^{3}}{\tau_{F}^{3}}, \\
& \mathbf{f}(t)=\left(3 \frac{t^{2}}{\tau_{F}^{2}}-2 \frac{t^{3}}{\tau_{F}^{3}}\right) \mathbf{f}_{F} .
\end{aligned}
$$

Alternatively, we have seen that the local counterdiabatic driving protocol [Eq. (55)] leads to the time evolution $\Phi(t)=\mathcal{U} \Psi(t)$. Imposing $\hat{\overline{\mathcal{H}}}=\hat{\mathcal{H}}_{0}$ at $t=\left\{0, \tau_{F}\right\}$ and demanding the initial and final states to be stationary, so that $\Phi=\Psi$, leads to Eqs. (72) and (73), supplemented by

$$
\ddot{\gamma}(0)=0, \quad \ddot{\gamma}\left(\tau_{F}\right)=0, \quad \ddot{\mathbf{f}}(0)=\mathbf{0}, \quad \ddot{\mathbf{f}}\left(\tau_{F}\right)=\mathbf{0},
$$

which are satisfied by an interpolating ansatz such as

$\gamma(t)=1+10\left(\gamma_{F}-1\right) \frac{t^{3}}{\tau_{F}^{3}}-15\left(\gamma_{F}-1\right) \frac{t^{4}}{\tau_{F}^{4}}+6\left(\gamma_{F}-1\right) \frac{t^{5}}{\tau_{F}^{5}}$,

$\mathbf{f}(t)=\left(10 \frac{t^{3}}{\tau_{F}^{3}}-15 \frac{t^{4}}{\tau_{F}^{4}}+6 \frac{t^{5}}{\tau_{F}^{5}}\right) \mathbf{f}_{F}$.

In effect, Eq. (76) provides a "recipe" of how to engineer shortcuts to adiabaticity in expansions and transport processes.

It is instructive to consider the amplitude $\chi(t)$ of the counterdiabatic term $\mathbf{F}=-\ddot{\mathbf{f}}_{F} \equiv \mathbf{f}_{F} \chi(t)$ to assist transport in terms of the dimensionless time $s \equiv t / \tau_{F}$ :

$$
\chi(t)=-60 s\left(2 s^{2}-3 s+1\right) \tau_{F}^{2} .
$$

We note that the function $\chi(t)<0$ in the interval $\left(0, \tau_{F} / 2\right)$, so that the CD term speeds up the wave packet toward the target position $\mathbf{f}_{F}$. In the subsequent stage $\left(\tau_{F} / 2, \tau_{F}\right)$, $\chi(t)>0$, so that the CD term decelerates the translation until the evolving state is centered at $\mathbf{q}=\mathbf{f}_{F}$ and the CD term vanishes. The extrema $\mathbf{F}_{ \pm}= \pm \mathbf{f}_{F} 45 / 8 \tau_{F}^{2}$ are reached at $t=\tau_{F} / 6(3 \pm \sqrt{3})$. For a transport function, $\mathbf{f}(t)=$ $f(t) \hat{\mathbf{n}}$ in the direction of an arbitrary unit vector $\hat{\mathbf{n}}$. Figure 2 shows the time dependence of both $f(t)$ and $\chi(t)$. CD provides an exact STA to the transport process, directly applicable to many-body systems such as trappedion chains, without the restrictions to perturbative treatments in previous proposals $[31,76]$. Alternative transport functions can be designed using optimal control theory [77].

The time dependence of the $\mathrm{CD}$ term to assist expansions scales in a similar way with the quench rate, that is, 

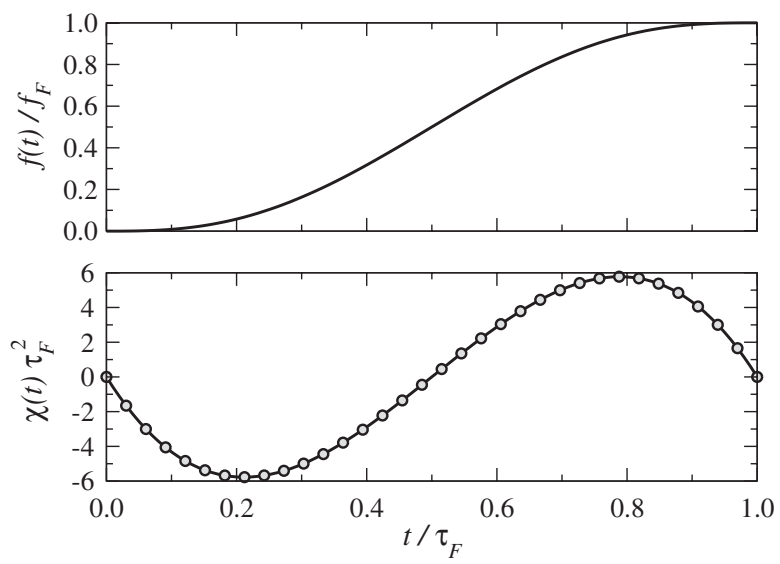

FIG. 2. Shortcut to adiabatic transport by local counterdiabatic driving. Whenever the trapping potential $U(\mathbf{q}, t)=$ $U_{0}[\mathbf{q}-f(t) \hat{\mathbf{n}}]$ [with $\gamma(t)=1$ ], the auxiliary counterdiabatic term with $\mathbf{F}=-\ddot{\mathbf{f}}_{F}=: \mathbf{f}_{F} \chi(t)$ induces the self-similar evolution $\Phi(t)=\mathcal{U} \Psi(t)$, which at $t=\left\{0, \tau_{F}\right\}$ reduces to $\Psi(t)=\Phi(t)$, and the auxiliary term vanishes. CD guarantees that the density profile of the system is centered at all times at $\mathbf{q}=f(t) \hat{\mathbf{n}}$. This protocol holds exactly for an arbitrary trapping potential $U_{0}(\mathbf{q})$ and is valid for an arbitrary single-particle, nonlinear, or many-body system, requiring no modulation of the coupling constant $\epsilon(t)$ in the case of interacting systems, i.e., $\epsilon(t)=1$.

$-m \ddot{\gamma} \overline{\mathbf{q}}_{i}^{2} / \gamma \propto \tau_{F}^{-2}$. In an early stage $\left(0, \tau_{F} / 2\right)$, the gain in the speed-up of the nonadiabatic expansion is provided by the auxiliary inverted harmonic potential, when $\ddot{\gamma}>0$; see Fig. 3. The quickly expanding cloud is then slowed down $(\ddot{\gamma}<0)$ by the $\mathrm{CD}$ term during the time interval $\left(\tau_{F} / 2, \tau_{F}\right)$, and it comes to rest as the CD term vanishes $\left(\ddot{\gamma} \rightarrow 0^{-}\right)$, and the completion of the process is approached as $t \rightarrow \tau_{F}$.
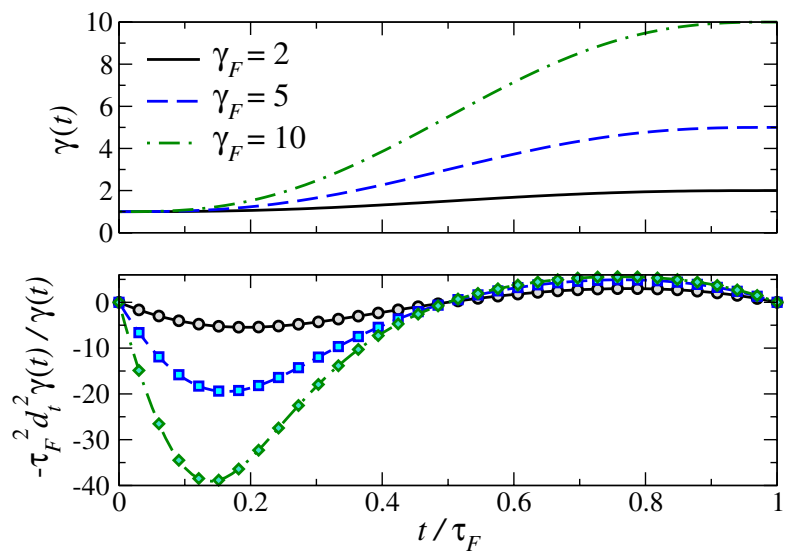

FIG. 3. Shortcut to an adiabatic expansion by local counterdiabatic driving. Whenever the trapping potential $U(\mathbf{q}, t)=$ $U_{0}[\mathbf{q} / \gamma(t)] / \gamma(t)^{2}$ [with $\mathbf{f}(t)=0$ ], the auxiliary counterdiabatic term modulated by $-m \ddot{\gamma} / \gamma(t)$ induces a nonadiabatic self-similar dynamics that reduces to the target state $\Psi(t)$ at $t=\left\{0, \tau_{F}\right\}$, when the auxiliary term vanishes. This protocol holds exactly for an arbitrary trapping potential $U_{0}(\mathbf{q})$ and can be applied to an arbitrary single-particle, nonlinear, or many-body system. In the case of interacting systems, it requires a modulation of the coupling constant $\epsilon(t)$, as in Eq. (50).
We point out that alternative trajectories can be designed that are optimal according to a variety of criteria, such as minimum mean energy or operating time with fixed resources, using optimal control theory. Stefanatos [78] has presented time-optimal protocols for the scale-invariant expansion dynamics in a time-dependent box. As we have seen, the auxiliary driving potential in a shortcut to an adiabatic expansion for this particular example [16] takes the general form associated with local CD protocols [28]. Hence, the results in Ref. [78] apply generally to CD scaleinvariant dynamics. Similarly, optimal trajectories for efficient transport [77] can be adopted for scale-invariant driving with $\mathbf{f}(t) \neq 0$. Furthermore, an analysis of the Ehrenfest dynamics [79] in CD protocols can be used as a guideline to engineer modulations of both $\gamma(t)$ and $\mathbf{f}(t)$.

\section{CONCLUDING REMARKS}

A unifying framework has been introduced to design shortcuts to adiabaticity in both classical and quantum systems for scale-invariant processes, such as expansions, compressions, and transport. The dynamical symmetry in these processes provides the leverage with respect to the original approach to counterdiabatic driving, which demands knowledge of the spectral properties of the instantaneous Hamiltonian of the system [20-22].

In particular, we found a closed-form expression for the auxiliary term in the counterdiabatic Hamiltonian and proposed a framework that allows us to rewrite this expression in local form, which is of relevance to experimental realizations. The formalism of generating functions provides a simple and intuitive way to find the canonical transformations that achieve this goal.

These findings were used to construct driving protocols mimicking adiabatic dynamics for multiparticle quantum systems with arbitrary trapping potentials, as illustrated in nonharmonic examples. As an upshot, the requirements to speed up finite-time thermodynamic processes are greatly loosened. We envision applications of these ideas in the development of optimal cooling schemes [80-84] and their experimental implementation [85]. Our results also facilitate the realization of friction-free quantum pistons $[16,35,78]$ and superadiabatic engines $[32,86]$ by relaxing the restrictions to the shape of the confining potential and the nature of the working medium.

Remarkably, these protocols are not restricted to noninteracting systems. In this context, we have illustrated the realization of shortcuts to adiabaticity in systems described by nonlinear equations of motion such as the Gross-Pitaevskii equation and higher-order nonlinear Schrödinger equations. By doing so, we have shown that it is possible to perform a fast counterdiabatic decompression (compression) of an interacting Bose-Einstein condensate in which the final state is free from excitations, providing a new route to the previous use of shortcuts to adiabaticity in the laboratory [87]. Further, we have shown 
that experimentally realizable counterdiabatic driving schemes can be applied to a wide variety of strongly correlated many-body systems, broadening the applicability of fast-transport single-particle protocols $[31,88]$ explored in trapped-ion experiments $[89,90]$, without resorting to the validity of perturbative methods. The applicability of shortcuts to adiabaticity to many-body quantum fluids paves the way to the realization of a quantum dynamical microscope, in which a quantum fluid cloud can be scaled up while preserving quantum correlations [15]. Spatially zooming up the quantum state in these systems constitutes an interesting complementary alternative to other imaging techniques based on long expansion times [70] and large numericalaperture optics $[91,92]$.

\section{ACKNOWLEDGMENTS}

It is a pleasure to thank Bogdan Damski, Iñigo L. Egusquiza, Emilie Passemar, Ayoti Patra, and Nikolai Sinitsyn for stimulating discussions and comments on the manuscript. S. D. and C. J. acknowledge support from the National Science Foundation (U.S.A.) under Grant No. DMR-1206971. This research is further supported by the U.S. Department of Energy through the LANL/ LDRD Program and a LANL J. Robert Oppenheimer Fellowship (A. d. C.).

\section{APPENDIX A: SCALE-INVARIANT EIGENFUNCTIONS}

In this Appendix, we prove the scaling law for wave functions used in Sec. II. Let $\psi_{n}^{0}(q)$ be an eigenfunction of the Hamiltonian $\hat{H}_{0}$ with

$$
\begin{aligned}
\hat{H}_{0}(1) \psi_{n}^{0}(q) & =E_{n} \psi_{n}^{0}(q), \\
\hat{H}_{0}(1) & =-\frac{\hbar^{2}}{2 m} \partial_{q}^{2}+U_{0}(q) ;
\end{aligned}
$$

then, $\psi_{n}(q, \gamma)=\alpha(\gamma) \psi_{n}^{0}(q / \gamma)$ is an eigenfunction of $\hat{H}_{0}(\gamma)$ with

$$
\hat{H}_{0}(\gamma)=-\frac{\hbar^{2}}{2 m} \partial_{q}^{2}+\frac{1}{\gamma^{2}} U_{0}(q / \gamma)
$$

where $\alpha(\gamma)$ is a normalization. The latter can be proven by direct evaluation. Consider

$$
\begin{aligned}
\hat{H}_{0}(\gamma) \psi_{n}(q, \gamma)= & -\frac{\hbar^{2}}{2 m} \partial_{q}^{2} \alpha(\gamma) \psi_{n}^{0}(q / \gamma) \\
& +\frac{1}{\gamma^{2}} U_{0}(q / \gamma) \alpha(\gamma) \psi_{n}^{0}(q / \gamma),
\end{aligned}
$$

which can be written as

$$
H_{0}(\gamma) \psi_{n}(q, \gamma)=\frac{\alpha(\gamma)}{\gamma^{2}} E_{n} \psi_{n}^{0}(\sigma)=\frac{E_{n}}{\gamma^{2}} \psi_{n}(q, \gamma),
$$

in terms of $\sigma=q / \gamma$. It follows that if $E_{n}$ is an eigenvalue of $H_{0}$, then $E_{n} / \gamma^{2}$ is an eigenvalue of $H_{0}(\gamma)$ [Eq. (A2)]. The prefactor $\alpha(\gamma)=\gamma^{-1 / 2}$ can be determined from the normalization of $\psi_{m}(q, \gamma)$ :

$$
\begin{aligned}
1 & =\int d q\left[\psi_{n}(q, \gamma)\right]^{2}, \\
& =[\alpha(\gamma)]^{2} \int d q\left[\psi_{n}(q / \gamma)\right]^{2}, \\
& =[\alpha(\gamma)]^{2} \gamma \int d \sigma\left[\psi_{n}(\sigma)\right]^{2}=[\alpha(\gamma)]^{2} \gamma .
\end{aligned}
$$

In the above Sec. II, we use a slightly more general result, where we allow additionally for transport along $q$. The validity can be easily checked by replacing $q \rightarrow q-f$ everywhere in the latter proof.

\section{APPENDIX B: ILLUSTRATIVE EXAMPLE: THE MORSE OSCILLATOR}

This Appendix is dedicated to an illustration of the classical results put forward in Ref. [33]. Recall the classical counterdiabatic Hamiltonian

$$
H(z, t)=H_{0}[z ; \lambda(t)]+\dot{\boldsymbol{\lambda}} \cdot \boldsymbol{\xi}[z, \lambda(t)],
$$

where $H_{1}(t)=\dot{\lambda} \cdot \xi[\lambda(t)]$ is the counterdiabatic term.

It is further shown that the generator $\xi$ satisfies

$$
\xi\left(z_{b} ; \lambda\right)-\xi\left(z_{a} ; \lambda\right)=\int_{a}^{b} d t \nabla \tilde{H}_{0}[z(t) ; \lambda]
$$

where $z_{a}$ and $z_{b}$ are two points on the same energy shell of $H_{0}(z, \lambda)$, and $z(t)$ is a trajectory that evolves under $H_{0}$ from $z_{a}$ to $z_{b}$. For the sake of simplicity of notation, we denote in the following the gradient with respect to the control parameter $\lambda$ by simply $\nabla$. In Eq. (B2), $\nabla \tilde{H}_{0}=\nabla H_{0}-$ $\left\langle\nabla H_{0}\right\rangle_{H_{0}, \lambda}$, where $\langle\ldots\rangle_{E, \lambda}$ is the microcanonical average with

$$
\langle\ldots\rangle_{E, \lambda} \equiv \frac{1}{\partial_{E} \Omega} \int d z \delta\left[E-H_{0}(z, \lambda)\right]
$$

Finally, $\Omega(E, \lambda)$ is the phase-space volume enclosed by the energy shell $E$

$$
\Omega(E, \lambda) \equiv \int d z \Theta\left[E-H_{0}(z, \lambda)\right]
$$

and we have

$$
\nabla E(\Omega, \lambda)=-\frac{\nabla \Omega(E, \lambda)}{\partial_{E} \Omega(E, \lambda)}=\left\langle\nabla H_{0}\right\rangle_{E, \lambda} .
$$

The latter formalism can be used to calculate an explicit expression for the generator $\xi$. 
We proceed by illustrating the above findings for a completely analytical solvable system, namely, the parametric Morse oscillator. The Morse potential can be written as [55]

$$
U(q)=U_{m}[\exp (-2 \beta q)-2 \exp (-\beta q)] .
$$

In the remainder of this Appendix, we compute $\xi$ [Eq. (B1)] explicitly for three different driving protocols. We start with scale-invariant driving, before we vary either only the potential width $\beta$ or the potential depth $U_{m}$.

\section{Scale-invariant parametrization} form

For scale-invariant driving $U(q)$, Eq. (B6) takes the

$$
U[q, \gamma(t)]=\frac{U_{m}}{\gamma^{2}(t)}\left[\exp \left(-2 \frac{\beta q}{\gamma(t)}\right)-2 \exp \left(-\frac{\beta q}{\gamma(t)}\right)\right] .
$$

For the sake of simplicity and to avoid clutter, we work in units where $U_{m}=1$ and $\beta=1$. In Fig. $4, U(q, \gamma)$ is shown for two different values of $\gamma$. For the present case, Eq. (B2) simplifies to read

$$
\xi\left(z_{b} ; \gamma\right)-\xi\left(z_{a} ; \gamma\right)=\int_{a}^{b} d t\left(\partial_{\gamma} H_{0}-\left\langle\partial_{\gamma} H_{0}\right\rangle\right)
$$

where $z$ is again the phase-space variable. In Fig. 5, we plot two energy shells corresponding to the same energy but different values of $\gamma$, whose volume is the microcanonical partition function $\Omega(E, \gamma)$.

As a first step, we have to calculate the phase-space volume $\Omega(E, \gamma)$, which is given by

$$
\begin{aligned}
\Omega(E, \gamma) & =\int_{q_{1}}^{q_{2}} d q p(q, E), \\
& =\int_{q_{1}}^{q_{2}} d q \sqrt{2 m[E-U(q, \gamma)]},
\end{aligned}
$$

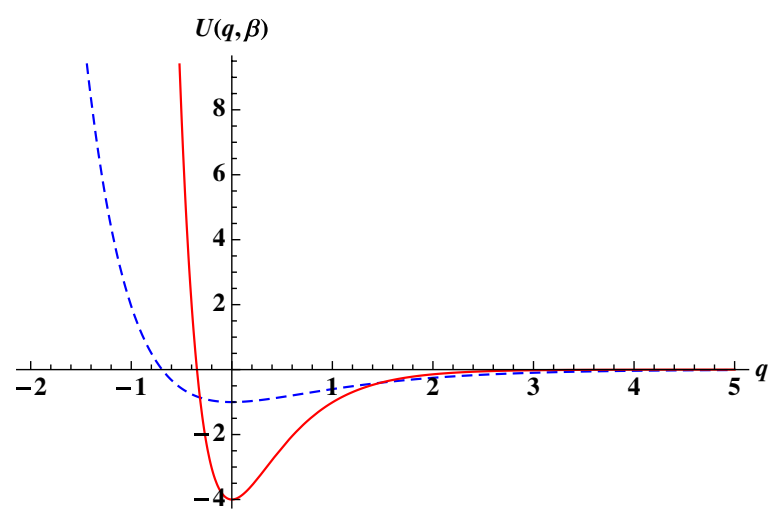

FIG. 4. Scale-invariant counterdiabatic driving of the Morse oscillator. Morse potential $U(q, \beta)$ [Eq. (B7)] for $\gamma=1$ (dashed blue line) and $\gamma=1 / 2$ (solid red line).

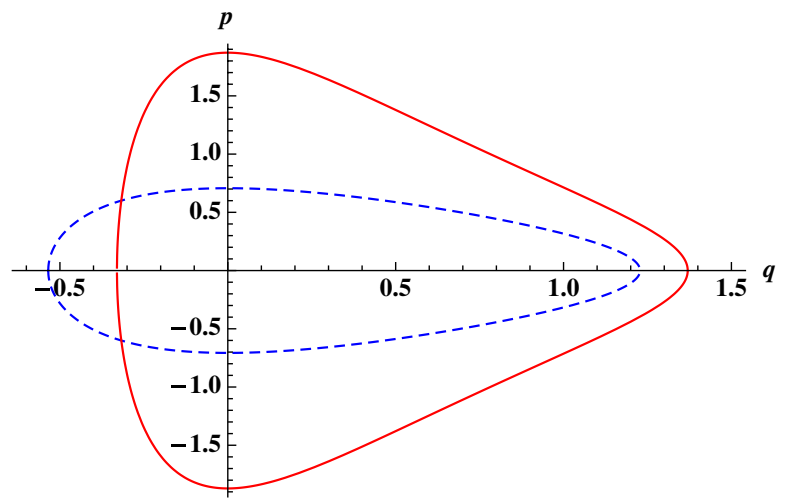

FIG. 5. Phase-space volume $\Omega(E, \gamma)$ corresponding to $U(q, \gamma)$ [Eq. (B7)] for $E=-1 / 2$ and $\gamma=1$ (dashed blue line) and $\gamma=1 / 2$ (solid red line).

where $p(q, E)$ is the momentum, and $p\left(q_{1,2}, E\right)=0$. Note that $\Omega(E, \gamma)$ is only finite for a bound state, which means that $0 \geq E \geq-\gamma^{-2}$. For the sake of simplicity, we continue in units, where the mass $m=1 / 2$. Then, the 0 s of the momentum are given by

$$
\begin{aligned}
& q_{1}=-\gamma \ln \left(1+\sqrt{1+E \gamma^{2}}\right), \\
& q_{2}=-\gamma \ln \left(1-\sqrt{1+E \gamma^{2}}\right) .
\end{aligned}
$$

The phase-space volume is obtained by calculating the integral in Eq. (B9). We have

$$
\Omega(E, \gamma)=2 \pi\left(1-\sqrt{-E \gamma^{2}}\right) .
$$

Accordingly, we obtain with Eq. (B8)

$$
\dot{\xi}=2 p^{2} / \gamma+2 q \gamma^{-4} \exp (-2 q / \lambda)-2 q \gamma^{-4} \exp (-q / \gamma) \text {. }
$$

Finally, $\xi$ is obtained by either integrating Eq. (B8) or by solving the partial differential equation

$$
\dot{\xi}=-\partial_{q} \xi \partial_{p} H_{0}+\partial_{p} \xi \partial_{q} H_{0}=-\left\{\xi, H_{0}\right\},
$$

where the curly brackets again denote the Poisson bracket. The right-hand side of Eq. (B13) can be explicitly written as

$$
\begin{aligned}
-\left\{\xi, H_{0}\right\}= & 2 p \partial_{q} \xi+2 \gamma^{-3} \exp (-2 q / \gamma) \partial_{p} \xi \\
& -2 \gamma^{-3} \exp (-q / \gamma) \partial_{p} \xi .
\end{aligned}
$$

One easily convinces oneself (almost by inspection) that a solution to Eq. (B13) is given by

$$
\xi=q p / \gamma
$$


The latter result is in perfect agreement with the above analysis that led to Eq. (21).

\section{Arbitrary parametrization}

Scale-invariant dynamics have proven to be theoretically useful, as they allow the computation of the counterdiabatic term $\xi$ in closed form. From an experimental point of view, however, it might be more relevant to vary the two parameters, the potential depth $U_{m}$ and the width $\beta$, independently. Therefore, we continue our discussion with examples where we vary only $U_{m}$ or $\beta$, while the other parameter is kept constant.

\section{a. Time-dependent width} form

We continue by considering a Morse potential of the

$$
U_{w}[q, \beta(t)]=\exp [-2 \beta(t) q]-2 \exp [-\beta(t) q],
$$

where we set again for the sake of simplicity $U_{m}=1$. This choice of units it not necessary but convenient, as it drastically reduces the clutter in the formulas. As before, we need to compute $\Omega(E, \beta)$ first. With the 0 s of the momentum $p(q, E)$ being given by

$$
\begin{aligned}
& q_{1}=-\frac{1}{\beta} \ln (1+\sqrt{1+E}), \\
& q_{2}=-\frac{1}{\beta} \ln (1-\sqrt{1+E}),
\end{aligned}
$$

we obtain

$$
\Omega_{w}(E, \beta)=2 \pi\left(\frac{1}{\beta^{2}}-\frac{\sqrt{-E}}{\beta}\right) .
$$

Accordingly, we have for $\dot{\xi}$

$$
\begin{aligned}
\dot{\xi}_{w}= & -2 p^{2} / \beta-2 \exp (-2 \beta q) / \beta+4 \exp (-\beta q) / \beta \\
& -2 q \exp (-2 \beta q)+2 q \exp (-\beta q) \\
& +4 \sqrt{-p^{2}-\exp (-2 \beta q)+2 \exp (\beta q)} / \beta^{2} .
\end{aligned}
$$

After a few lines, a simplified expression for $\xi$ can be found, which reads

$$
\begin{aligned}
\xi_{w}= & \frac{p}{\beta^{2}}+\frac{q p}{\beta}-\frac{1}{\beta^{2}} \arctan \left(\frac{1-\exp (-\beta q)}{p}\right) \\
& -\frac{2}{\beta^{3}} \operatorname{arccot}\left(\frac{p \exp (-\beta q) \sqrt{-H_{0}(q, p)}}{1+\exp (\beta q) H_{0}(q, p)}\right) .
\end{aligned}
$$

We observe that the second term in Eq. (B18) is identical to the counterdiabatic term for scale-invariant dynamics. Moreover, $H_{0}$ is the "unperturbed" Hamiltonian as a function of the phase-space variables $p$ and $q$. Note also that the latter expression is valid for all bound states, i.e., $E=H_{0}(q, p) \leq 0$.

\section{b. Time-dependent depth}

As a final example, let us consider a Morse potential, whose depth $U_{m}$ is varied while its width is kept constant. We have

$$
U_{d}\left[q, U_{m}(t)\right]=U_{m}(t)[\exp (-2 q)-2 \exp (-q)],
$$

where, this time, we set $\beta=1$. As before, we need the 0 s of the momentum, which read here

$$
\begin{aligned}
& q_{1}=\ln \left(\frac{-U_{m}+\sqrt{U_{m}\left(E+U_{m}\right)}}{E}\right) \text { and } \\
& q_{2}=\ln \left(-\frac{U_{m}+\sqrt{U_{m}\left(E+U_{m}\right)}}{E}\right),
\end{aligned}
$$

with which we obtain

$$
\Omega_{d}\left(E, U_{m}\right)=2 \pi\left(\sqrt{U_{m}}-\sqrt{-E}\right) .
$$

In complete analogy to the previous two examples, we compute $\dot{\xi}$, which reads here

$$
\begin{aligned}
\dot{\xi}_{d}= & \exp (-2 q)-2 \exp (-q) \\
& +\sqrt{-p^{2}-U_{m}[\exp (-2 q)+2 \exp (q)]} / \sqrt{U_{m}} .
\end{aligned}
$$

Although the expression for $\dot{\xi}$ appears to be simpler, the integral $\xi$ is much more involved. After several lines of simplification, we obtain

$\xi_{d}=-\frac{p}{2 U_{m}}-\frac{1}{2 \sqrt{U_{m}}} \arctan \left(\frac{p \exp (q)\left\{\exp (q) H_{0}+U_{m}+[\exp (q)-1] \sqrt{-U_{m} H_{0}}\right\}}{\exp (2 q) \sqrt{-H_{0}^{3}}+\exp (q)[\exp (q)-1] \sqrt{U_{m}} H_{0}+[1-2 \exp (q)] U_{m} \sqrt{-H_{0}}+[\exp (q)-1] \sqrt{U_{m}^{3}}}\right)$.

By comparing the closed-form expressions for the counterdiabatic terms in Eqs. (B15), (B20), and (B25), it becomes obvious how much scale-invariant driving simplifies the situation. Although for scale-invariant driving $H_{1}$ can be brought into local form with the help of an appropriate coordinate transformation, this simplification seems hardly feasible in the general case. 
[1] P. G. Collins, A. Zettl, H. Bando, A. Thess, and R. E. Smalley, Nanotube Nanodevice, Science 278, 100 (1997).

[2] X. M. H. Huang, C. A. Zorman, M. Mehregany, and M. L. Roukes, Nanoelectromechanical Systems: Nanodevice Motion at Microwave Frequencies, Nature (London) 421, 496 (2003).

[3] B.E. Kane, A Silicon-Based Nuclear Spin Quantum Computer, Nature (London) 393, 133 (1998).

[4] T. Kinoshita, T. Wenger, and D. S. Weiss, A Quantum Newton's Cradle, Nature (London) 440, 900 (2006).

[5] J.E. Avron, A. Raveh, and B. Zur, Adiabatic Quantum Transport in Multiply Connected Systems, Rev. Mod. Phys. 60, 873 (1988).

[6] P. Král, I. Thanopulos, and M. Shapiro, Coherently Controlled Adiabatic Passage, Rev. Mod. Phys. 79, 53 (2007).

[7] V. Giovannetti, S. Lloyd, and L. Maccone, Quantum Metrology, Phys. Rev. Lett. 96, 010401 (2006).

[8] B. Andresen, P. Salamon, and R. S. Berry, Thermodynamics in Finite Time, Phys. Today 37, No. 9, 62 (1984).

[9] A. Trabesinger, Quantum Simulation, Nat. Phys. 8, 263 (2012).

[10] M. A. Nielsen and I. L. Chuang, Quantum Computation and Quantum Information (Cambridge University Press, Cambridge, England, 2000).

[11] A. Messiah, Quantum Mechanics (Wiley, New York, 1966).

[12] E. Torrontegui, S. S. Ibáñez, S. Martínez-Garaot, M. Modugno, A. del Campo, D. Guéry-Odelin, A. Ruschhaupt, X. Chen, and J. G. Muga, Chapter 2-Shortcuts to Adiabaticity, Adv. At. Mol. Opt. Phys. 62, 117 (2013).

[13] X. Chen, A. Ruschhaupt, S. Schmidt, A. del Campo, D. Guéry-Odelin, and J. G. Muga, Fast Optimal Frictionless Atom Cooling in Harmonic Traps: Shortcut to Adiabaticity, Phys. Rev. Lett. 104, 063002 (2010).

[14] X. Chen, E. Torrontegui, and J. G. Muga, Lewis-Riesenfeld Invariants and Transitionless Quantum Driving, Phys. Rev. A 83, 062116 (2011).

[15] A. del Campo, Frictionless Quantum Quenches in Ultracold Gases: A Quantum-Dynamical Microscope, Phys. Rev. A 84, 031606(R) (2011).

[16] A. del Campo and M. G. Boshier, Shortcuts to Adiabaticity in a Time-Dependent Box, Sci. Rep. 2, 648 (2012).

[17] S. Masuda and K. Nakamura, Fast-Forward of Adiabatic Dynamics in Quantum Mechanics, Proc. R. Soc. A 466, 1135 (2009).

[18] S. Masuda and K. Nakamura, Acceleration of Adiabatic Quantum Dynamics in Electromagnetic Fields, Phys. Rev. A 84, 043434 (2011).

[19] E. Torrontegui, S. Martínez-Garaot, A. Ruschhaupt, and J. G. Muga, Shortcuts to Adiabaticity: Fast-Forward Approach, Phys. Rev. A 86, 013601 (2012).

[20] M. Demirplak and S. A. Rice, Adiabatic Population Transfer with Control Fields, J. Phys. Chem. A 107, 9937 (2003).

[21] M. Demirplak and S. A. Rice, Assisted Adiabatic Passage Revisited, J. Phys. Chem. B 109, 6838 (2005).

[22] M. Berry, Transitionless Quantum Driving, J. Phys. A 42, 365303 (2009).

[23] M. V. Berry, Quantal Phase Factors Accompanying Adiabatic Changes, Proc. R. Soc. A 392, 45 (1984).
[24] M. Demirplak and S. A. Rice, On the Consistency, Extremal, and Global Properties of Counterdiabatic Fields, J. Chem. Phys. 129, 154111 (2008).

[25] A. del Campo, M. M. Rams, and W. H. Zurek, Assisted Finite-Rate Adiabatic Passage across a Quantum Critical Point: Exact Solution for the Quantum Ising Model, Phys. Rev. Lett. 109, 115703 (2012).

[26] M. G. Bason, M. Viteau, N. Malossi, P. Huillery, E. Arimondo, D. Ciampini, R. Fazio, V. Giovannetti, R. Mannella, and O. Morsch, High-Fidelity Quantum Driving, Nat. Phys. 8, 147 (2011).

[27] J. Zhang, J. H. Shim, I. Niemeyer, T. Taniguchi, T. Teraji, H. Abe, S. Onoda, T. Yamamoto, T. Ohshima, J. Isoya, and D. Suter, Experimental Implementation of Assisted Quantum Adiabatic Passage in a Single Spin, Phys. Rev. Lett. 110, 240501 (2013).

[28] A. del Campo, Shortcuts to Adiabaticity by Counterdiabatic Driving, Phys. Rev. Lett. 111, 100502 (2013).

[29] A. Mostafazadeh, Dynamical Invariants, Adiabatic Approximation and the Geometric Phase (Nova, New York, 2001).

[30] J. G. Muga, X. Chen, S. Ibáñez, I. Lizuain, and A. Ruschhaupt, Transitionless Quantum Drivings for the Harmonic Oscillator, J. Phys. B 43, 085509 (2010).

[31] E. Torrontegui, S. Ibáñez, X. Chen, A. Ruschhaupt, D. Guéry-Odelin, and J. G. Muga, Fast Atomic Transport without Vibrational Heating, Phys. Rev. A 83, 013415 (2011).

[32] J. Deng, Q. Wang, Z. Liu, P. Hänggi, and J. Gong, Boosting Work Characteristics and Overall Heat Engine Performance via Accelerated Adiabatic Control: Quantum and Classical, Phys. Rev. E 88, 062122 (2013).

[33] C. Jarzynski, Generating Shortcuts to Adiabaticity in Quantum and Classical Dynamics, Phys. Rev. A 88, 040101(R) (2013).

[34] P. J. Gambardella, Exact Results in Quantum Many-Body Systems of Interacting Particles in Many Dimensions with $\overline{S U}(1,1)$ as the Dynamical Group, J. Math. Phys. (N.Y.) 16, 1172 (1975).

[35] S. Di Martino, F. Anzá, P. Facchi, A. Kossakowski, G. Marmo, A. Messina, B. Militello, and S. Pascazio, A Quantum Particle in a Box with Moving Walls, J. Phys. A 46, 365301 (2013).

[36] Hamiltonians that include products of space and momentum operators $q$ and $p$ are nonlocal, whereas local Hamiltonians contain only terms that depend on at most sums of $q$ and $p$.

[37] H. Goldstein, Classical Mechanics (Addison-Wesley, Reading, MA, 1959).

[38] I. H. Duru, Quantum Treatment of a Class of TimeDependent Potentials, J. Phys. A 22, 4827 (1989).

[39] M. V. Berry and G. Klein, Newtonian Trajectories and Quantum Waves in Expanding Force Fields, J. Phys. A 17, 1805 (1984).

[40] H. R. Lewis, Class of Exact Invariants for Classical and Quantum Time-Dependent Harmonic Oscillators, J. Math. Phys. (N.Y.) 9, 1976 (1968).

[41] H. R. Lewis and W. B. Riesenfeld, An Exact Quantum Theory of the Time-Dependent Harmonic Oscillator and 
of a Charged Particle in a Time-Dependent Electromagnetic Field, J. Math. Phys. (N.Y.) 10, 1458 (1969).

[42] N. J. Günther and P. G. L. Leach, Generalized Invariants for the Time-Dependent Harmonic Oscillator, J. Math. Phys. (N.Y.) 18, 572 (1977).

[43] P. G. L. Leach, Invariants and Wavefunctions for Some Time-Dependent Harmonic Oscillator-Type Hamiltonians, J. Math. Phys. (N.Y.) 18, 1902 (1977).

[44] P. G. L. Leach, On a Direct Method for the Determination of an Exact Invariant for the Time-Dependent Harmonic Oscillator, J. Aust. Math. Soc. Series B, Appl. Math. 20, 97 (1977).

[45] P. G. L. Leach, On the Theory of Time-Dependent Linear Canonical Transformations as Applied to Hamiltonians of the Harmonic Oscillator Type, J. Math. Phys. (N.Y.) 18, 1608 (1977).

[46] M. A. Lohe, Exact Time Dependence of Solutions to the Time-Dependent Schrödinger Equation, J. Phys. A 42, 035307 (2009).

[47] V. A. Yurovsky, M. Olshanii, and D. S. Weiss, Collisions, Correlations, and Integrability in Atom Waveguides, Adv. At. Mol. Opt. Phys. 55, 61 (2008).

[48] K. Huang, Bose Condensate in External Potential: A Thomas-Fermi Approach, arXiv:cond-mat/9609033.

[49] A. M. Perelomov, The Simple Relation between Certain Dynamical Systems, Commun. Math. Phys. 63, 9 (1978).

[50] E. Timmermans, P. Tommasini, M. Hussein, and A. Kerman, Feshbach Resonances in Atomic Bose-Einstein Condensates, Phys. Rep. 315, 199 (1999).

[51] K. Staliunas, S. Longhi, and G. J. de Valcárcel, Faraday Patterns in Low-Dimensional Bose-Einstein Condensates, Phys. Rev. A 70, 011601 (2004).

[52] A. del Campo, Fast Frictionless Dynamics as a Toolbox for Low-Dimensional Bose-Einstein Condensates, Europhys. Lett. 96, 60005 (2011).

[53] M. Moshinsky and Y. F. Smirnov, The Harmonic Oscillator in Modern Physics (Harwood Academic, Amsterdam, 1996).

[54] K. Henderson, C. Ryu, C. MacCormick, and M. G. Boshier, Experimental Demonstration of Painting Arbitrary and Dynamic Potentials for Bose-Einstein Condensates, New J. Phys. 11, 043030 (2009).

[55] S. Flügge, Practical Quantum Mechanics (Springer, Heidelberg, 1971), Vol. I.

[56] F. Cooper, A. Khare, and U. Sukhatme, Supersymmetry and Quantum Mechanics, Phys. Rep. 251, 267 (1995).

[57] Y. Castin and R. Dum, Bose-Einstein Condensates in Time Dependent Traps, Phys. Rev. Lett. 77, 5315 (1996).

[58] Yu. Kagan, E. L. Surkov, and G. V. Shlyapnikov, Evolution of a Bose Gas in Anisotropic Time-Dependent Traps, Phys. Rev. A 55, R18 (1997).

[59] I. L. Egusquiza, M. Modugno, and M. A. Valle Basagoiti, Multiple-Scale Approach for the Expansion Scaling of Superfluid Quantum Gases, Phys. Rev. A 84, 043629 (2011).

[60] A. Kundu, Integrable Nonautonomous Nonlinear Schrödinger Equations Are Equivalent to the Standard Autonomous Equation, Phys. Rev. E 79, 015601(R) (2009).

[61] L. D. Fadeev and L. A. Takhtajan, Hamiltonian Methods in the Theory of Solitons (Springer, Heidelberg, 2007).
[62] E. B. Kolomeisky, T. J. Newman, J. P. Straley, and Xiaoya Qi, Low-Dimensional Bose Liquids: Beyond the GrossPitaevskii Approximation, Phys. Rev. Lett. 85, 1146 (2000).

[63] E. B. Kolomeisky and J. P. Straley, Renormalization-Group Analysis of the Ground-State Properties of Dilute Bose Systems in d Spatial Dimensions, Phys. Rev. B 46, 11749 (1992).

[64] M. D. Girardeau, Relationship between Systems of Impenetrable Bosons and Fermions in One Dimension, J. Math. Phys. (N.Y.) 1, 516 (1960).

[65] M. D. Girardeau and E. M. Wright, Breakdown of TimeDependent Mean-Field Theory for a One-Dimensional Condensate of Impenetrable Bosons, Phys. Rev. Lett. 84, 5239 (2000).

[66] The exact many-body quantum system describing a TonksGirardeau gas ( $N$ one-dimensional bosons with contact interactions of infinite amplitude) is exactly solvable by means of the Bose-Fermi duality as discussed by Girardeau [64]. It is remarkable that the classical integrability condition in terms of the zero-curvature representation for the mean-field Kolomeisky equation describing this system has not yet been found.

[67] Y. E. Kim and A. L. Zubarev, Density-Functional Theory of Bosons in a Trap, Phys. Rev. A 67, 015602 (2003).

[68] B. Damski, Shock Waves in Ultracold Fermi (Tonks) Gases, J. Phys. B 37, L85 (2004).

[69] Z. Ozcakmakli and C. Yuce, Shortcuts to Adiabaticity for Growing Condensates, Phys. Scr. 86, 055001 (2012).

[70] G. Lamporesi, S. Donadello, S. Serafini, F. Dalfovo, and G. Ferrari, Spontaneous Creation of Kibble-Zurek Solitons in a Bose-Einstein Condensate, Nat. Phys. 9, 656 (2013).

[71] C. N. Weiler, T. W. Neely, D. R. Scherer, A. S. Bradley, M. J. Davis, and B. P. Anderson, Spontaneous Vortices in the Formation of Bose-Einstein Condensates, Nature (London) 455, 948 (2008).

[72] A. del Campo, T. W. B. Kibble, and W. H. Zurek, Causality and Non-equilibrium Second-Order Phase Transitions in Inhomogeneous Systems, J. Phys. Condens. Matter 25, 404210 (2013).

[73] A. del Campo and W. H. Zurek, Universality of Phase Transition Dynamics: Topological Defects from Symmetry Breaking, arXiv:1310.1600.

[74] V. Gritsev, P. Barmettler, and E. Demler, Scaling Approach to Quantum Non-equilibrium Dynamics of Many-Body Systems, New J. Phys. 12, 113005 (2010).

[75] J. G. Muga, X. Chen, A. Ruschhaupt, and D. Guéry-Odelin, Frictionless Dynamics of Bose-Einstein Condensates under Fast Trap Variations, J. Phys. B 42, 241001 (2009).

[76] M. Palmero, E. Torrontegui, D. Guéry-Odelin, and J. G. Muga, Fast Transport of Two Ions in an Anharmonic Trap, Phys. Rev. A 88, 053423 (2013).

[77] X. Chen, E. Torrontegui, D. Stefanatos, J.-S. Li, and J. G. Muga, Optimal Trajectories for Efficient Atomic Transport without Final Excitation, Phys. Rev. A 84, 043415 (2011).

[78] D. Stefanatos, Optimal Shortcuts to Adiabaticity for a Quantum Piston, Automatica 49, 3079 (2013).

[79] S. Choi, R. Onofrio, and B. Sundaram, Ehrenfest Dynamics and Frictionless Cooling Methods, Phys. Rev. A 88, 053401 (2013). 
[80] P. Salamon, K. H. Hoffmann, Y. Rezek, and R. Kosloff, Maximum Work in Minimum Time from a Conservative Quantum System, Phys. Chem. Chem. Phys. 11, 1027 (2009).

[81] K. H. Hoffmann, P. Salamon, Y. Rezek, and R. Kosloff, Time-Optimal Controls for Frictionless Cooling in Harmonic Traps, Europhys. Lett. 96, 60015 (2011).

[82] S. Choi, R. Onofrio, and B. Sundaram, Optimized Sympathetic Cooling of Atomic Mixtures via Fast Adiabatic Strategies, Phys. Rev. A 84, 051601 (2011).

[83] P. Salamon, K. H. Hoffmann, and A. Tsirlin, Optimal Control in a Quantum Cooling Problem, Appl. Math. Lett. 25, 1263 (2012).

[84] C. Yuce, Fast Frictionless Expansion of an Optical Lattice, Phys. Lett. A 376, 1717 (2012).

[85] J.-F. Schaff, X.-L. Song, P. Vignolo, and G. Labeyrie, Fast Optimal Transition between Two Equilibrium States, Phys. Rev. A 82, 033430 (2010).

[86] A. del Campo, J. Goold, and M. Paternostro, More Bang for Your Buck: Towards Super-adiabatic Quantum Engines, arXiv:1305.3223.

[87] J.-F. Schaff, X.-L. Song, P. Capuzzi, P. Vignolo, and G. Labeyrie, Shortcut to Adiabaticity for an Interacting Bose-Einstein Condensate, Europhys. Lett. 93, 23001 (2011).
[88] A. Negretti, A. Benseny, J. Mompart, and T. Calarco, Speeding Up the Spatial Adiabatic Passage of Matter Waves in Optical Microtraps by Optimal Control, Quantum Inf. Process. 12, 1439 (2013).

[89] R. Bowler, J. Gaebler, Y. Lin, T. R. Tan, D. Hanneke, J. D. Jost, J. P. Home, D. Leibfried, and D. J. Wineland, Coherent Diabatic Ion Transport and Separation in a Multizone Trap Array, Phys. Rev. Lett. 109, 080502 (2012).

[90] A. Walther, F. Ziesel, T. Ruster, S. T. Dawkins, K. Ott, M. Hettrich, K. Singer, F. Schmidt-Kaler, and U. Poschinger, Controlling Fast Transport of Cold Trapped Ions, Phys. Rev. Lett. 109, 080501 (2012).

[91] W. S. Bakr, J. I. Gillen, A. Peng, S. Fölling, and M. Greiner, A Quantum Gas Microscope for Detecting Single Atoms in a Hubbard-Regime Optical Lattice, Nature (London) 462, 74 (2009).

[92] M. Endres, M. Cheneau, T. Fukuhara, C. Weitenberg, P. Schau, C. Gross, L. Mazza, M. C. Bañuls, L. Pollet, I. Bloch, and S. Kuhr, Observation of Correlated ParticleHole Pairs and String Order in Low-Dimensional Mott Insulators, Science 334, 200 (2011). 